APPLICATION OF RAY ACOUSTICS TO THE PROPAGATION OF SOUND IN DUCTS CONTAINING SHEARED FLOWS

by

Denny Warren Grimm

Thesis submitted to the Graduate Faculty of the Virginia Polytechnic Institute and State University in partial fullfillment of the requirements for the degree of

MASTER OF SCIENCE

in

Mechanical Engineering

APPROVED:

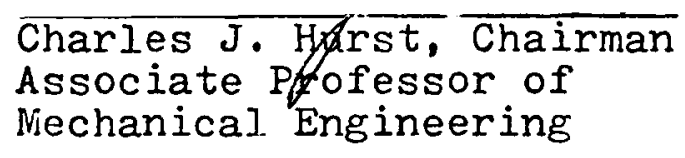

Larry D. Mitchell Associate Professor of Mechanical Engineering
Harry H. Robertshaw Assistant Professor of Mechanical Engineering

November, 1972 


\section{ACKNOWLEDGEMENTS}

This research is part of the acoustic research program at Virginia Polytechnic Institute and State University.

The author sincerely thanks for

his kind understanding, patience and helpful suggestions throughout the research and the preparation of this thesis. He also wishes to thank and

for serving on his advisory committee. Finally, he wishes to thank his wife for typing the final copy of the thesis and making this endeavor possible with her encouragement. 


\section{TABLE OF CONTENTS}

Page

Introduction .............. 1

Background Information ......... 3

Normal Mode Approach. ........ 3

Geometric Acoustics . . . . . . . 5

Description of Sound Propagation

Model ............. 8

Flow Duct . . . . . . . . . 8

Flow Profile Model.......... 8

Sound Source............ 12

Ray Tracing Equations. . . . . . . . 14

Intensity Loss Equations . . . . . . . 20

Expression for Spreading Loss . . . . . 20

Spreading Loss Equations. . . . . . 24

Conditions at a Reflecting Boundary . . . . 25

Calculation of Total Intensity Loss. . . . . . 27

Validity of Ray Acoustics Approach . . . . . 32

Discussion of Results. . . . . . . . 38

Ray Plots . . . . . . . . 38

Intensity Loss Plots........ . 50

Conclusions. . . . . . . . . . . 64

References . . . . . . . . . 66

Appendix . . . . . . . . . . 69

vita.............. . . 70 


\section{IIST OF FIGURES AND TABLES}

Figure

$\underline{\text { Page }}$

1 Duct Model Geometry . . . . . . 9

2 Typical Duct Flow Profiles. . . . . 10

3 Iine Sound Source Model . . . . . . 13

4 Wave Propagating Through Constant Flow

Velocity Region... . . . . . 15

5 Phase Velocity and Ray Velocity Directions

in a Moving Medium. . . . . . . . 17

6 Side View of an Infinitesimal Ray-

Bundle. . . . . . . . . . 23

7 A Typical Set of Ray Faths. . . . . 28

8 Horizontal Distances Required to Define

a Ray Path. . . . . . . . . 30

9 Ray Diagram for Upstream Propagation,

$\mathrm{CLM}=0.3 . \mathrm{BLT}=0.3 \ldots . . . .40$

10 Ray Diagram for Upstream Fropagation,

CLM $=0.5 . \mathrm{BLT}=0.3 . . . . . .44$

11 Ray Diagram for Upstream Propagation,

CLM $=0.7$, BLT $=0.3 . . . . . .44$

12 Ray Diagram for Downstream Propagation,

$\mathrm{CLM}=0.5 . \mathrm{BLT}=0.1 . . . . . .44$

13 Ray Diagram for Downstream Propagation,

CLM $=0.5$, BLT $=0.2 . . . . . . .45$

14 Ray Diagram for Downstream Propagation, CLM $=0.5$, BLT $=0.3 . . . . . . .46$

15 Ray Diagram for Downstream Propagation,

$\mathrm{CLM}=0.3, \mathrm{BLT}=0.3 . . . . . .447$

16 Ray Diagram for Downstream Propagation, $\mathrm{CLM}=0.7 . \mathrm{BLT}=0.3 . . . . . .448$

17 Downstream Intensity Loss for CLM $=0.5$, $\mathrm{BLT}=0.1, \mathrm{XDES}=1.0, \mathrm{~F}=10.0 . . .52$ 
18 Downstream Intensity Loss for CLM $=0.5$, $\mathrm{BIT}=0.1, \mathrm{XDES}=1.0, \mathrm{~F}=20.0 . . .53$

19 Downstream Intensity Loss for CLM $=0.5$, $\mathrm{BLT}=0.2, \mathrm{XDES}=1.0, \mathrm{~F}=10.0 \ldots . .54$

20 Downstream Intensity Loss for CLM $=0.5$, BIT $=0.2, \quad$ XDES $=1.0, F=20.0 . . .55$

21 Downstream Intensity Loss for CLM $=0.5$, $\mathrm{BLT}=0.3, \mathrm{XDES}=1.0, \mathrm{~F}=10.0 . . .56$

22 Downstream Intensity Loss for CLM $=0.5$, $\mathrm{BLT}=0.3, \mathrm{XDES}=1.0, \mathrm{~F}=20.0 . . .57$

23 Downstream Intensity Loss for $C L M=0.5$, $\mathrm{BLT}=0.4, \mathrm{XDES}=1.0, \mathrm{~F}=10.0 . . .58$

24 Downstream Intensity Loss for $C L M=0.5$. BLT $=0.4$, XDES $=1.0, F=20.0 . . .59$

25 Downstream Intensity Loss for CLM $=0.3$, $\mathrm{BLT}=0.3, \mathrm{XDES}=1.0, \mathrm{~F}=10.0 . . .60$

26 Downstream Intensity Loss for $\mathrm{CLM}=0.3$, $\mathrm{BLT}=0.3, \mathrm{XDES}=1.0, \mathrm{~F}=20.0 . . .61$

27 Downstream Intensity Loss for CLM $=0.7$, $\mathrm{BLT}=0.3, \mathrm{XDES}=1.0, \mathrm{~F}=10.0 . . .62$

28 Downstream Intensity Loss for CLM $=0.7$, $\mathrm{BLT}=0.3, \mathrm{XDES}=1.0, \mathrm{~F}=20.0 . . .63$

1 Maximum Wavelength for Valid Application of Ray Acoustics. . . . 36 


\section{NOMENCLATURE}
A
Ray-bundle area
$A_{0}$
Reference ray-bundle area
c
Speed of sound
d
Boundary-layer thickness
e
Energy density
f
Actual frequency
Nondimensional frequency parameter
h
Verticle position in duct
$\mathrm{H}$
Duct height
I
Ray intensity
Io
Reference ray intensity
k
Ray path curvature
I
Duct length
Nondimensional duct length
m
Arbitary integer number
M
Mach number
$\mathrm{M}_{\mathrm{CI}}$
Centerline Mach number
n
Wavefront unit normal
$\mathrm{N}$
Spreading loss
$\mathrm{P}$
Pressure amplitude
$r$
Radius
R
Ray path cycle distance
$\mathrm{R}_{1}$
Horizontal distance to receiver height intercept
$\mathrm{R}_{2}$
Horizontal distance to wall intercept
$\mathbf{S}$
Path arc length 


\begin{tabular}{|c|c|}
\hline t & Actual time \\
\hline $\mathrm{T}^{*}$ & Nondimensional time \\
\hline$\overline{u_{n}}$ & Phase velocity vector \\
\hline$\overline{u_{r}}$ & Group velocity vector \\
\hline$\overline{\mathrm{V}}$ & Flow velocity vector \\
\hline W & Wavefront arc length \\
\hline $\mathrm{x}$ & Horizontal Spreading Loss Variable \\
\hline $\mathbf{x}$ & Actual horizontal coordinate \\
\hline$x^{*}$ & Nondimensional horizontal coordinate \\
\hline $\mathbf{y}$ & Actual width coordinate \\
\hline $\mathrm{z}$ & Verticle spreading loss variable \\
\hline $\mathbf{z}$ & Actual verticle coordinate \\
\hline$z^{*}$ & Nondimensional verticle coordinate \\
\hline$\Theta$ & Angular spreading loss variable \\
\hline$\theta$ & Wave normal angle \\
\hline$\theta_{0}$ & Initial wave normal angle \\
\hline$\delta$ & Wavelength \\
\hline$\mu$ & Index of refraction \\
\hline p & Density \\
\hline$\rho_{0}$ & Static density \\
\hline$\phi$ & Ray angle \\
\hline
\end{tabular}




\section{INTRODUCTION}

In this investigation the effects of a sheared flow on a sound field were studied using a geometric or ray acoustics approach. Differential equations describing the ray paths and ray spreading losses in a homogeneous moving medium were developed and solved numerically to make intensity predictions at specified cross sections of the hard walled duct.

The use of ray acoustics as a possible tool for studying the effects of flow and flow gradients on sound levels in ducts has long been recognized but has not been utilized. This has been because of the inaccuracy of ray theory for lower frequency ranges. Normal mode theory is valid for both low and high frequencies so most studies have used that approach and have only alluded to the possibilities of a ray approach. Ray theory is much simpler than normal mode theory, and the results obtained provide an excellent physical representation of the sound field.

Discrete frequency sound in ducts can result from aerodynamic loading on turbine or compressor blades or from rotorstator interaction. This noise occurs at the blade passage frequency which equals the product of the shaft rotational speed and the number of blades. To effectively reduce the resulting radiated noise, treatment of the exhaust and inlet ducts with absorptive linings is imperative. In the selection of the best materials and the optimum locations for duct treat- 
ment the influence of the flow field can not be ignored. The flow field can have significant effects on both the attenuation characteristics of the liner and on the sound field itself. Analytical treatments of this problem are very involved because of the many complicating physical effects which modify the sound field. A complete treatment of the problem would have to include not only the effects of flow and flow gradients, but also complex and nonlinear acoustic wall impedance, variable duct geometry, finite duct length, and modal energy distribution. 


\section{BACKGROUND INFORMATION}

\section{A. Normal Mode Results}

In the past the influence of a flowing medium on the propagation of sound in ducts has been developed through the normal mode approach. The case of a duct with a uniform flow has been treated by Ingard (1), Tack and Lambert (2), Eversman (3), Morfey (4), Doak and Vaidya (5), and others.

Pridmore-Brown (6) was the first to solve the wave equation with the inclusion of both uniform flow and flow gradient effects for sound propagating between two plane parallel walls. Assuming zero viscosity, Pridmore-Brown showed the basic equation describing this situation to be

$$
\frac{1}{c^{2}} \frac{d^{2} p}{d t^{2}}=\left(1-M^{2}\right) \frac{d^{2} p}{d x^{2}}+\frac{d^{2} p}{d y^{2}}-\frac{2 M}{c} \frac{d^{2} p}{d x d t}+{ }^{2} \rho_{0} c \frac{d M}{d y} \frac{d v}{d x}
$$

where

$$
\begin{aligned}
& \mathrm{C}=\text { speed of sound } \\
& \mathrm{P}=\text { pressure amplitude } \\
& M=\text { Mach number } \\
& \mathrm{v}=\text { particle velocity } \\
& P_{0}=\text { static density of medium }
\end{aligned}
$$

To solve this equation the wavenumber eigenvalues must be found which give nontrivial results and which also satisfy the wall boundary conditions. The boundary conditions are complex in value except for the case of hard walled ducts. Pridmore-Brown solved this equation for the downstream propagation of the lowest order mode in the shear layer for both constant gradient and one-seventh power-law flow profiles. 
His solution for downstream propagation predicted an increased sound pressure level near the hard walls due to refraction of the sound wave by the sheared flow. The effects of refraction were found to be more pronounced at higher frequencies and higher Mach numbers. PridmoreBrown also found that a flowing medium increases the local or phase velocity of a sound wave propagating downstream. This phenomena is called convection. It has the effect of decreasing the usefulness of the absorptive wall liners because the wave passes through the lined duct in less time and is thus less attenuated. The solution obtained by Pridmore-Brown did not hold near the duct walls for the turbulent flow case. Mungur and Gladwell ( 7 ) developed a numerical solution to find the eigenvalues to the wave equation. Their results agreed quite well with Pridmore-Brown's solution at points not near the duct wall and was valid at points near the duct wall as well. The effects of a flowing medium have also been confirmed experimentally in work performed by Mechel, Mertens, and Schilz (8). For downstream propagation, they found a frequency below which attenuation decreased with flow (convection effect) and above which attenuation increased with flow (refraction effect). For upstream propagation, convection effects caused increased attenuation and refraction effects caused decreased attenuation just opposite to the downstream case.

- Mungur and Plumblee (9) modeled the flow in a cylindrical 
duct with a constant velocity core and a boundary-layer near the walls. The velocity profile chosen for the boundary-layer was one quarter of a sine wave. Their objective was to study the effect of varying the shear-layer thickness. For downstream propagation the effects of sound refraction were found to increase with increasing boundary-layer thickness and were found to cause higher sound pressure levels near the duct walls. Eversman (10) found, however, that this refraction of sound for downstream propagation was less significant than for upstream propagation. Eversman also showed a pronounced decrease in acoustic wall liner performance due to upstream refraction.

Hersh, Beranek, and Newman (II) concluded that the effects of boundary-layer refraction became significant when the ration of boundary-layer thickness to acoustic wavelength was equal to or greater than unity.

In another study on boundary-layer refraction, Savkar (12) also concluded that refraction effects can not be ignored for the high Mach numbers and high frequencies typically encountered in turbojet engines.

\section{B. Geometric Acoustics}

Ray acoustics theory for a stationary medium can be found in many references $(13,14,15,16)$. In the past, its use has been confined largely to the study of the propagation of sound in the ocean, where the speed of sound is a function 
of temperature, salinity, and changes in pressure associated with changes in depth. More recently, the ray approach has been used to study the propagation of sound through the atmosphere in studying the sonic boom problem, as discussed by Hayes, et. al. (17) and Buell (18).

The concept of rays and ray-tube area can be traced back to Lord Rayleigh in 1878 (19). Barton (20), in 1901 was the first to distinquish between wave normals and rays for a moving medium and to give the correct planar ray tracing equations. The theory for a moving medium was subsequently formalized by Heller (21), Milne (22), and Kornhauser (23) in work which resulted in generalized derivations of the eikonal equation and Snell's law. Emden (24) identified the rays in terms of energy transport, but Blokhintsev (25) was responsible for deriving the expression for energy density:

$$
e=\frac{p^{2}}{2 p_{0} c^{2}} \cdot \frac{\text { phase velocity }}{\text { sound velocity }}
$$

where

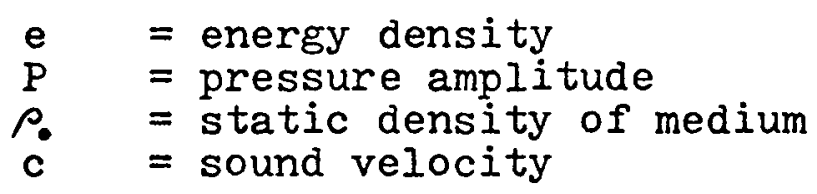

He proved that the acoustic energy flowed in the direction of the ray paths and that the intensity of the sound equaled the energy density multiplied by the ray velocity.

Molor and Soloman (26) derived a method for calculating the intensity loss along a ray path called the "ray-bundle approach." This procedure involved the calculation of two 
adjacent ray paths which were initially separated by a small angle. The distance between the rays was calculated as the rays propagated. This method was acceptable if the rays remained close to each other, but for long-range calculations and for reflections of the rays from a surface the method failed.

A better method was developed by Anderson, Gocht, and Sirota (27) which expressed the spreading loss at a point of interest on a ray in terms of spatial derivatives of the position vector from the sound source to the point. This method had the distinct advantage of being applicable to rays which had experienced many reflections from a boundary. Ugincius (28) extended this method to include the case where the index of refraction varied in two directions in an inhomogeneous medium. 


\section{DESCRIPTION OF SOUND PROPAGATION MODEL}

\section{A. Flow Duct}

The duct model employed for this study was equivalent to a pair of parallel flat plates of infinite length and width. This model is applicable to the study of sound propagation in an annular duct with radius of curvature which is much greater than the duct height. The walls of the duct were assumed to be perfectly rigid and smooth. The ray equations were developed for this model with reference to a rectangular coordinate system oriented as shown in Fig. 1 . The duct dimensions were nondimensionalized with respect to duct height. The temperature in the duct was assumed to be uniform and all viscous absorption by the medium was neglected due to the short propagation distances involved. The effects of impedance changes at the end of this finite duct and the resulting reflected waves caused by impedance mismatch were also neglected in this study.

\section{B. Flow Profile Model}

The flow model used was two-dimensional and consisted of a potential core in the duct center attached to a boundarylayer of constant thickness near the duct walls (See Fig. 2). This type of flow profile is a very realistic simulation of the flow field expected in an aircraft engine exhaust or inlet duct. Fully developed flow is not possible because the ducts 


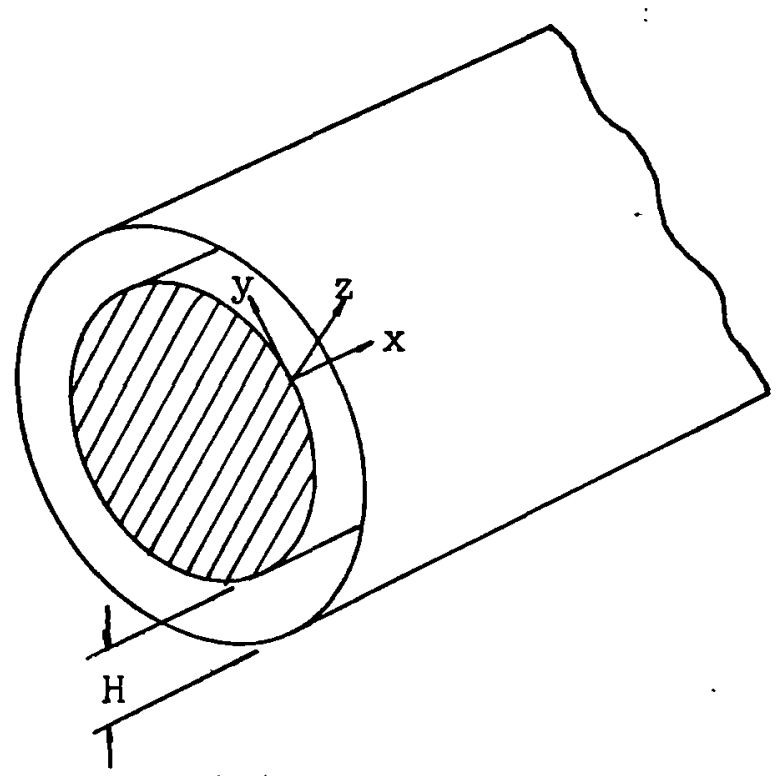

(a) Annular Duct

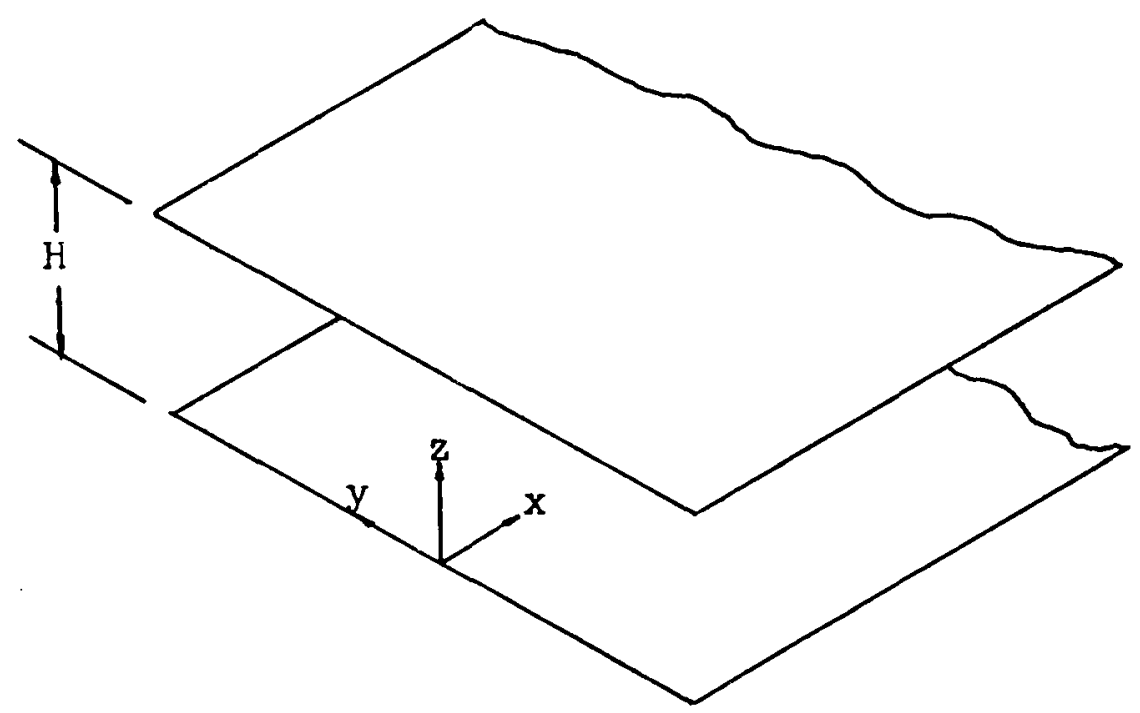

(b) Straight Duct

Fig. 1 Duct Model Geometry 


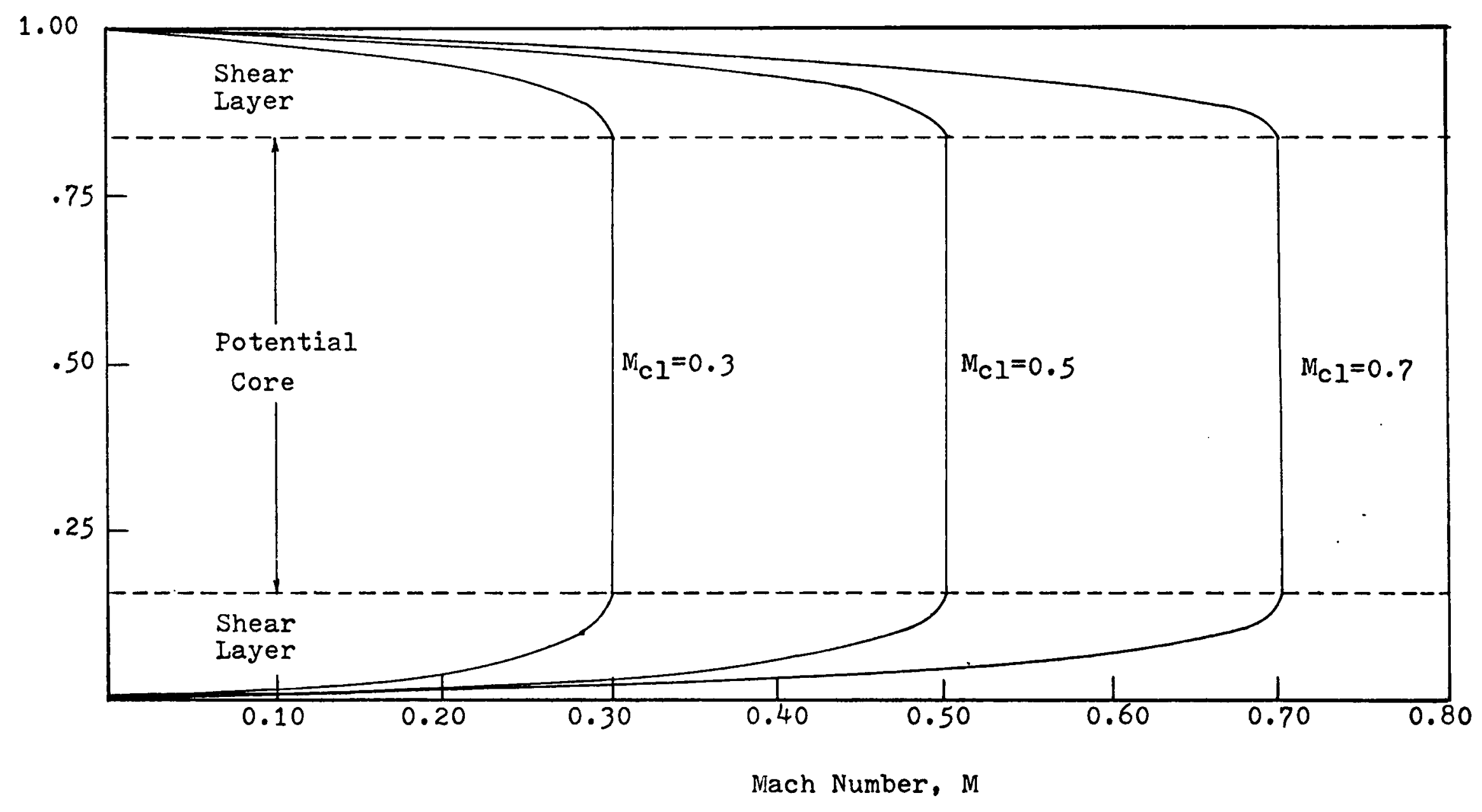

Fig. 2 Typical Duct Flow Profiles 
of aircraft engines are not long enough to allow complete boundary-layer growth. The flow model used here was very similar to the one used by Mungur and Plumblee (9) and by Hersh, Beranek, and Newman (II) except that they simulated the constant boundary-layer as one quarter of a sine wave.

The curvature of the sound rays is dependent upon the gradient of the flow field, and the spreading of the rays is dependent upon the spatial changes in the flow gradient. For these reasons it was a necessary requirement that the first and second spatial derivatives of the flow field be defined and continuous functions at all points across the duct. The equation for the shear layer must have first and second spatial derivatives which vanish at the interface between the potential flow core and the boundary-layer and which remain finite at points very near the duct wall. None of the shear layer models typically found in the literature could satisfy these criteria. To solve this difficulty a power-law flow model was developed which fulfilled these requirements and which made the numerical calculations possible. The equations for this flow profile and its derivatives can be written as

$$
\begin{gathered}
M(z)=M_{c l} \quad I-\left[(d)^{-m}(z-d)^{m}\right] \\
\frac{d M(z)}{d z}=(m)\left(M_{c l}\right)(d)^{-m}(z-d)^{m-1} \\
\frac{d^{2} M(z)}{d z}=(m)(m-I)\left(M_{c l}\right)(d)^{-m}(z-d)^{m-2}
\end{gathered}
$$


Various flow profiles were considered by specifying the centerline Mach number and boundary-layer thickness. By experimentation, a realistic profile was achieved by using an integer power $m$ of 4. Typical profiles based on this exponent have been showed in Fig. 2 for different values of centerline Mach number and a boundary-layer thickness of 0.2 .

\section{Sound Source}

The sound source in the duct was assumed to be a pulsating line parallel to the $\mathrm{y}$-axis with all points pulsating in phase. The wave generated by this source is cylindrical and expands uniformly in all radial directions when not diffracted by a flow field. This choice for the source can be justified from a physical standpoint by considering the pressure pulsations radiated by a row of blades in an annular duct. If the radius of curvature of the annular duct is much greater than the duct height, then the arc formed by points on the blades can be approximated by a straight line. Without the presence of a flow field the rays will originate at points on the line source and travel in directions normal to the expanding cylindrical wave as shown in Fig. 3a. In the presence of a sheared flow the rays do not remain straight, but are bent due to diffraction effects in the $x-z$ plane as shown in Fig. 3b. When the rays become bent the intensity of the wave will no longer be inversely proportional to the distance from the source and a more sophisticated means for calculating intensity is required. 


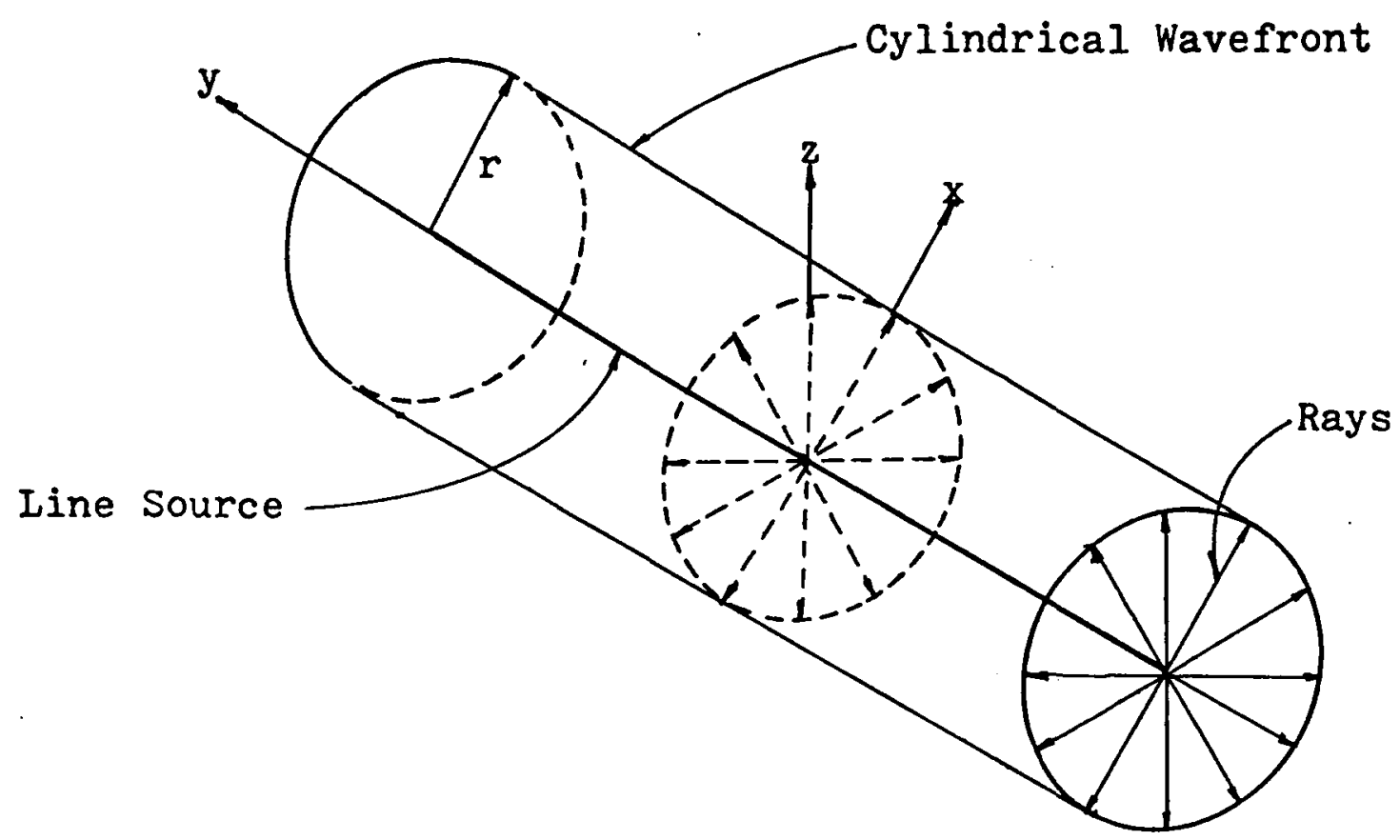

(a) No Flow

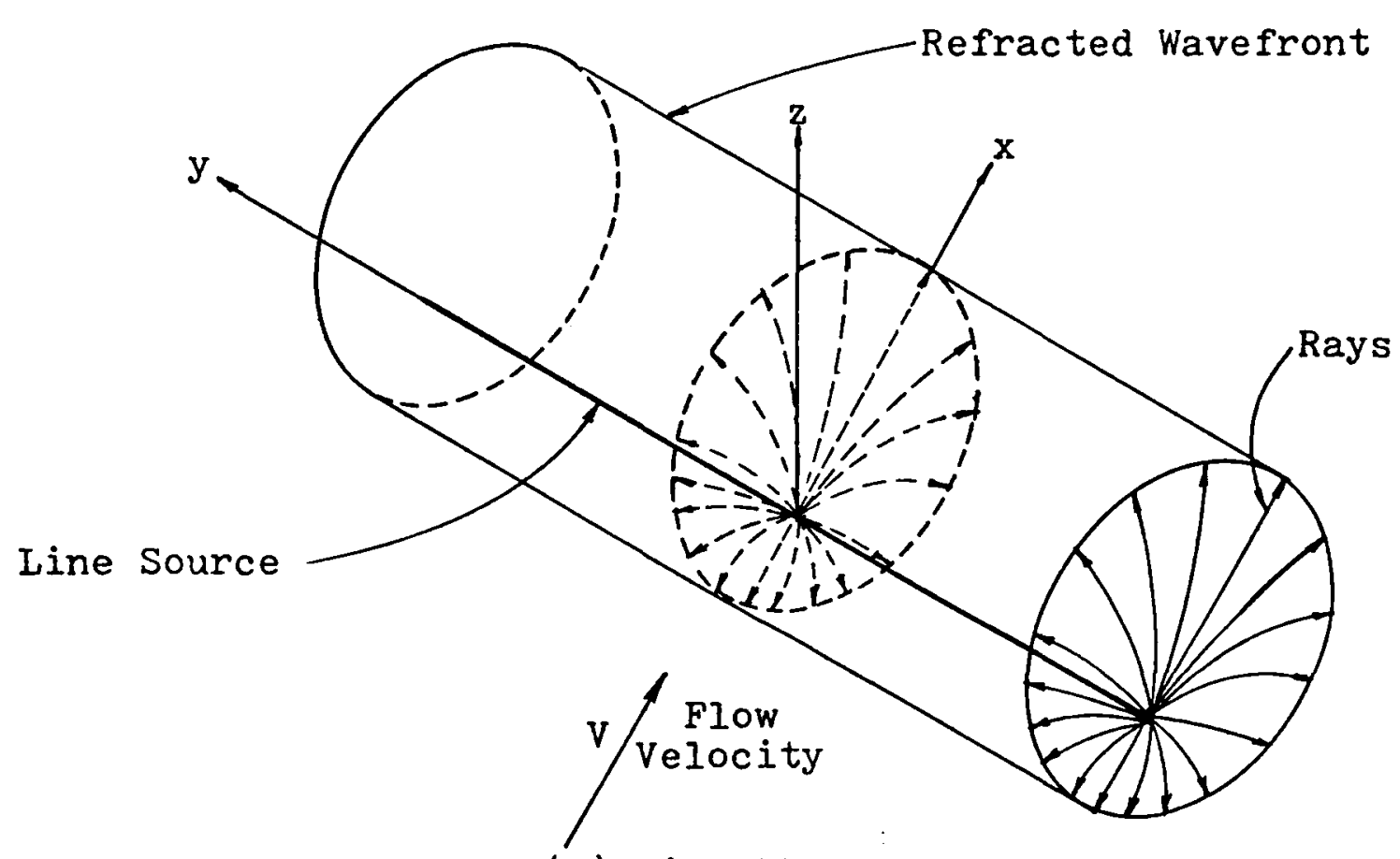

(b) With Flow

Fig. 3 Line Sound Source Model 


\section{RAY TRACING EQUATIONS}

A set of coupled, nonlinear, differential equations must be derived which described the ray paths through the moving medium. These paths represent the trajectories along which the acoustic energy of the sound wave is flowing. It is important to the understanding of geometric acoustics that the relationships between the ray paths, the wavefront, and the wave normals be presented. In Fig. 4, a wavefront which is propagating through a constant flow velocity zone(velocity $=\mathrm{V}$ ) is represented by the line $A B$ at some instant of time $t=t_{0}$. Huyghen's principle states that every point along the wavefront $A B$ will act as a simple source which radiates uniformly in every direction. The envelope of all these "Huyghen wavelets" originating along line $A B$ will then define the new wavefront at some later instant of time $t_{l}$. The wavelet which originates from point $A$ will expand a distance $c \Delta t$ in the time increment and will also translate horizontally a distance of $V A t$. Line $A C$ is then defined as the ray direction and also the direction of energy flow. This direction will in general always be different from the ray normal direction, but there does exist a geometrical relationship between the two. With the flow velocity in terms of Mach number the relationship between wave normal angle $\theta$ and ray angle $\phi$ can be written as

$$
\cos \theta=\cos \phi\left[1-M(z)^{2} \sin ^{2} \phi\right]^{\frac{1}{2}}-M(z) \sin ^{2} \phi .
$$




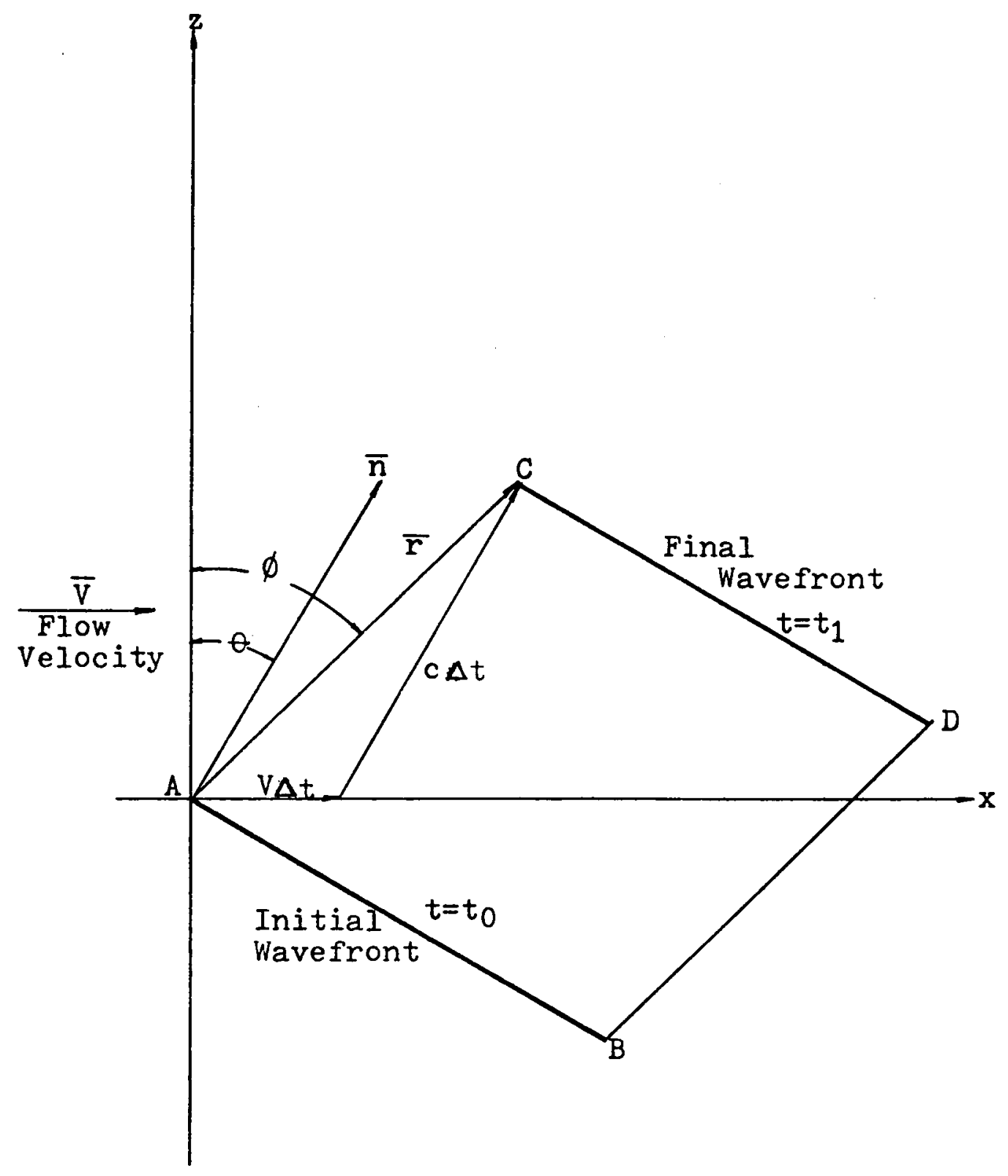

Fig. 4 Wave Propagating Through Constant Flow Velocity Region 
Two other definitions necessary to geometric acoustics are those for phase velocity and group velocity. Figure 5 shows a wavefront which is propagating in a medium where the flow velocity is represented by the vector $\vec{V}$. Phase velocity is defined as the velocity with which the wave expands in the normal direction. In a still medium the phase velocity is equal to the speed of sound. In a moving medium this velocity can be written as the sum of the speed of sound and the dot product of the flow velocity vector $\bar{V}$ and the unit normal vector $\overline{\mathrm{n}}$ :

$$
\overline{u_{n}}=[c+\bar{n} \cdot \bar{v}] \bar{n} \text {. }
$$

The group velocity is defined as the speed at which the wave travels in the ray direction. From Fig. 5, this group or ray velocity is seen to equal the vector sum of the normal speed of sound vector $\bar{n} c$ and the flow velocity vector $\bar{V}$ and can be written as

$$
\overline{u_{r}}=c \bar{n}+\bar{v}
$$

With this background it is now possible to write equations defining the ray paths directly from the definition of group velocity. The group velocity is written in terms of its coordinate components to yield

$$
\begin{aligned}
& \frac{d x}{d t}=\left(\overline{u_{r}}\right)_{x}=c \cos \theta+v \\
& \frac{d z}{d t}=\left(\overline{u_{r}}\right)_{z}=c \sin \theta .
\end{aligned}
$$

Along with these equations it is also necessary to 


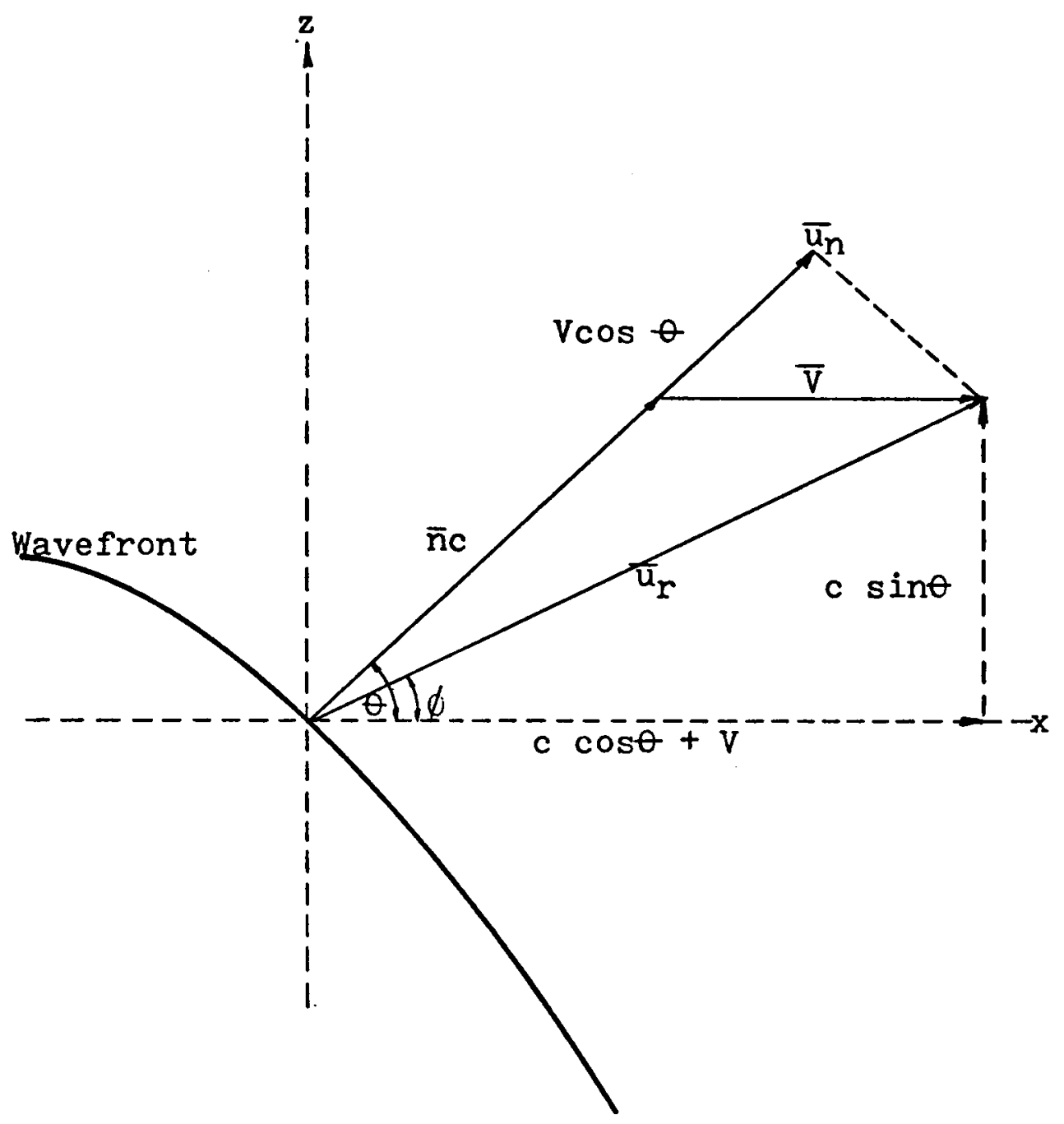

Fig. 5 Phase Velocity and Ray Velocity Directions in a Moving Medium 
know how the wave normal vector $\overline{\mathrm{n}}$ changes along the ray path. The refraction law for the wave normal in its most general form has derived in the literature see Hayes (17) and can be written as

$$
\frac{-d \bar{n}}{d t}=\nabla c+(\nabla \bar{v}) \cdot \bar{n}-\bar{n}[\bar{n} \cdot \nabla c+\bar{n} \cdot(\nabla \nabla) \cdot \bar{n}] .
$$

In the present study the speed of sound is assumed constant across the duct, and the flow velocity $\bar{V}$ is a function of $z$ only. With these conditions, the general equation 8 reduces to

$$
\frac{-d \bar{n}}{d t}=\frac{d V(z)}{d z} \bar{k} \cdot \bar{n}-\bar{n}\left(\bar{n} \cdot \frac{d V(z)}{d z} \bar{k} \cdot \bar{n}\right) \quad
$$

Since $\bar{n}$ is a unit normal vector it can be written in terms of its components as

$$
\bar{n}=\bar{i} \cos \theta+\bar{k} \sin \theta \quad .
$$

Making use of this substitution equation 9 can be simplified to the following result:

$$
\frac{d \theta}{d t}=-\cos ^{2} \theta \frac{d V(z)}{d z} \quad .
$$

The solutions for $x, z$, and $\theta$, from equations $7 a, 7 b$, and 11 , completely describe the path trajectories of the sound rays through a boundless medium.

The medium under study is bounded, however, by the walls of the duct. At those surfaces the assumption was made of total reflection of the ray with no phase change. Therefore, the only change in the ray trace variables was a sign change of the ray normal angle since the angle of reflection equaled 
the angle of incidence. With these assumptions the following conditions at the boundary hold:

$$
\Delta \mathrm{x}=0 \quad \Delta \mathrm{z}=0 \quad \Delta \theta=-2 \theta \quad .
$$

Each ray path can then be uniquely defined by specifying an initial source position $\left(x_{0}, z_{0}\right)$ and ray normal angle, $\theta_{0}$.

In order to minimize the number of physical parameters, the following nondimensional quantities were used:

$$
\begin{aligned}
& M=\mathrm{V} / \mathrm{c}, \text { Mach number; } \\
& \mathrm{T}^{*}=\mathrm{tc} / \mathrm{H}, \text { nondimensional time; } \\
& \mathrm{I}^{*}=\mathrm{L} / \mathrm{H}, \text { nondimensional duct length; } \\
& \mathrm{F}^{*}=\mathrm{fH} / \mathrm{c}, \text { nondimensional frequency parameter; } \\
& \mathrm{x}^{*}=\mathrm{x} / \mathrm{H}, \text { nondimensional } \mathrm{x} \text { coordinate; } \\
& \mathrm{z}^{*}=\mathrm{z} / \mathrm{H}, \text { nondimensional } \mathrm{z} \text { coordinate: }
\end{aligned}
$$

Therefore, the ray trace system of equations can be rewritten in a nondimensional form as

$$
\begin{aligned}
& \frac{d x^{*}}{d T^{*}}=\cos \theta+M\left(z^{*}\right) \\
& \frac{d z^{*}}{d T^{*}}=\sin \theta \\
& \frac{d \theta}{d T^{*}}=-\cos ^{2} \theta \frac{d M\left(z^{*}\right)}{d z}
\end{aligned}
$$




\section{INTENSITY LOSS EQUATIONS}

\section{A. Expression for Spreading Loss $\mathrm{N}$}

The average rate of energy flow through a unit area of the wavefront is defined as intensity. Variations in the wave phase velocity can cause refraction of the ray paths, and the area through which energy flows may not increase uniformly and may even decrease with distance. The acoustic power transmitted at any point along a bundle of rays, however, is constant and will equal the product of the intensity $I$ and the ray-bundle area $A$. Thus the intensity at any point along the ray path can be related to a reference intensity at a point near the sound source by the following relationship:

$$
I_{O A}=I A \quad \text { A }
$$

Spreading loss accounts for the changes in intensity associated with these changes in area along the ray path, and it can be calculated directly from ray geometry. The spreading loss of a ray-bundle is defined as

$$
N=-10 \log \left(I / I_{O}\right)=-10 \log \left(A_{O} / A\right) d B .
$$

Thus the problem of calculating intensity loss along a ray path becomes a geometric problem stated in terms of ray-tube area.

The ray-tube area can be calculated for two rays which are separated by a very small differential angle $d \theta_{0}$. The 
procedure does not involve the calculation of two ray paths, however, because the differential angle is allowed to approach zero in the limit. The ray-bundle area is calculated along the path of a single ray even though an isolated ray can not possess a physical area. Because of this the ray-tube area can be considered as a finite measure of a differential area along each ray. It is not physically identifiable on the ray diagram.

The set of differential equations required to calculate spreading loss were derived through partial differentiation of the ray-trace equations. This differentiation was performed with respect to $\theta_{0}$, the initial angle of the wave normal. The approach follows one developed by G. Anderson et al. (27) for an inhomogeneous still medium. It is applied here to a homogeneous moving medium.

Consider two rays separated by a small initial angle difference as shown in Fig. 6. At a reference radius $r_{0}$ very close to the source refraction effects are negligible. The ray paths within this small radius will be straight and the geometric spreading can be considered cylindrical. The ray-bundle area at this radius for a unit-width slice of the wavefront will be, therefore,

$$
d A_{0}=r_{0} d \theta_{0}
$$

The intensities at all points along the ray paths were calculated as spreading losses or gains from the intensity at this reference area. 


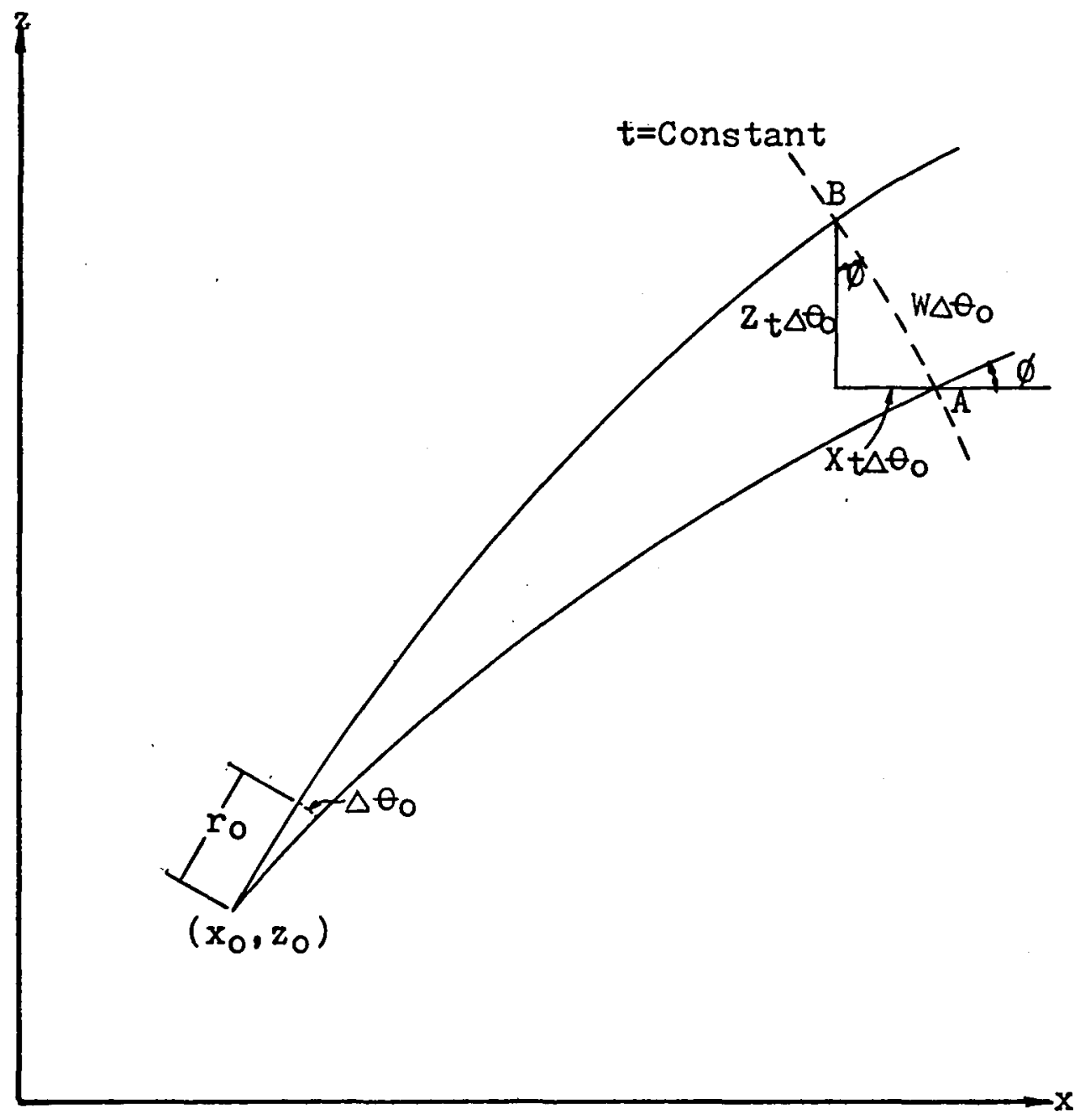

Fig. 6 Side View of an Infinitesimal Ray Bundle 
Now consider points $A$ and $B$ which lie on adjacent rays at points of equal phase. To calculate the ray-bundle area, it is necessary to calculate the arc length of the wavefront's xz-plane intercept connecting these points. At points of equal time the location of points $A$ and $B$ become functions of another independent variable besides time, the initial wave normal angle $\theta_{0}$. For rays distinquished by a small initial angle change, points of equal phase will be separated in relation to the derivative of the ray-trace variables ( $x$, z) with respect to the initial wave normal angle. This gives rise to a set of spreading loss variables which are defined as follows:

$$
X=\left(\frac{d x}{d \theta_{0}}\right)_{t} \quad z=\left(\frac{d z}{d \theta_{0}}\right)_{t} \quad \Theta=\left(\frac{d \theta}{d \theta_{0}}\right)_{t}
$$

Another variable closely related to these is the corresponding change in arc length of the wavefront with respect to $\theta_{0}$ which is symbolized by

$$
w=\left(\frac{d w}{d \theta_{0}}\right)_{t}
$$

where $w=$ ray-bundle arc length. From the geometry shown in Fig. 6, this arc length variable can be written in terms of the variable $Z$ as

$$
W=2 / \cos \phi \quad .
$$

When the solution of equation 19 is known the ray-tube area for a unit slice of the wave can be written as

$$
\mathrm{dA}=W \mathrm{~d} \theta_{0} \cdot
$$


The spreading loss along the ray path can then be calculated by the use of equation 15 as

$$
\begin{gathered}
N=-10 \log \left(d A_{0} / d A\right)=-10 \log \left(r_{0} / W\right) . \\
\text { B. Spreading Loss Equations }
\end{gathered}
$$

The procedure used for calculating spreading loss required solving for the variables defined by equations 17 . To arrive at the set of spreading loss differential equations, use was made of the following relation:

$$
\frac{d}{d \theta_{0}}\left(\frac{d s}{d t}\right)=\frac{d}{d t}\left(\frac{d s}{d \theta_{0}}\right)=\frac{d S}{d t}
$$

where $S$, which represents the derivative with respect to $\theta_{0}$, was replaced by $x, y$, and $\theta$ successively. Differentiation of the ray-trace system of equations with respect to $\theta_{0}$ yielded the following:

$$
\begin{gathered}
\frac{d X}{d t}=-\sin \theta \Theta+\frac{d M(z)}{d z} z \\
\frac{d z}{d t}=\cos \theta \Theta \\
\frac{d \Theta}{d t}=\frac{-d^{2} M(z)}{d^{2} z} z \cos ^{2} \theta+2 \frac{d M(z)}{d z} \cos \theta \sin \theta \Theta .
\end{gathered}
$$

The initial conditions can be calculated by evaluating $x^{*}\left(t^{*}\right), y^{*}\left(t^{*}\right)$, and $\theta\left(t^{*}\right)$ at $t^{*}=0$ and then differentiating with respect to $\theta_{0}$. Performing this differentiation gives the required initial conditions for the spreading loss system:

$$
x(0)=0 \quad z(0)=0 \quad \theta(0)=1 .
$$

The solutions to the spreading loss equations $22 \mathrm{a}$ through $22 \mathrm{c}$, 
along with the ray trace equations $13 a$ through $13 \mathrm{c}$, furnished enough information to calculate spreading loss at any point on a given ray for a boundless medium with a continuous index of refraction.

\section{Conditions at a Reflecting Boundary}

The conditions for a horizontal boundary have been derived by Ugincius (28). In his derivation, however, the independent ray path parameter was arc length rather than travel time. The ray paths being curves in space are dependent upon the parameters chosen, whereas the spreading loss being a scalar point function, is independent of the ray parameters as proven by Warfield and Jacobson(29). Likewise, the reflection conditions for the spreading loss equations are also scalar point functions which are independent of the ray parameters. The reflection conditions can be derived from first principles and shown to be invariant with the ray parameters chosen (see Appendix). The procedure used in this derivation was identical to that used by Ugincius except that travel time was an independent variable instead of arc length.

The conditions at a horizontal reflecting boundary were found to be

$\Delta \mathrm{x}_{t}=0 \quad \Delta \mathrm{z}_{t}=-2 \mathrm{z}_{\mathrm{t}} \quad \Delta \Theta_{t}=2\left[\frac{(\mathrm{k})}{\mathrm{dz} / \mathrm{dt}} \mathrm{z}_{\mathrm{t}}-\Theta_{\mathrm{t}}\right]$

where $k$ is the ray path curvature.

The curvature of the ray path can be evaluated from its parametric form as (see reference 30 ) 


$$
k=\frac{\frac{d z^{*}}{d t^{*}} \frac{d^{2} x^{*}}{d t^{* 2}}-\frac{d x^{*}}{d t^{*}} \frac{d^{2} z^{*}}{d t^{*}}}{\left[\left(\frac{d x^{*}}{d t^{*}}\right)^{2}+\left(\frac{d z^{*}}{d t^{*}}\right)^{2}\right]^{3 / 2}}
$$

where $x^{*}$ and $z^{*}$ are the ray trace variables (see equations 13). Performing the indicated differentiations and simplifying, equation 25 yields the following:

$$
k=\frac{\frac{d \theta}{d t}[1+M(z) \cos \theta]}{\left[1+2 \cos \theta M(z)+M^{2}(z)\right] 3 / 2}
$$

The reflection conditions were evaluated at the duct wall where $M(z)=0$; therefore, at the duct wall

$$
k=\frac{d \theta}{d t}=\frac{d M(z)}{d z} \cdot(\cos \theta)^{2}
$$

Equations 24 can now be written as

$$
\Delta \mathrm{x}_{\mathrm{t}}=0, \quad \Delta \mathrm{z}_{\mathrm{t}}=-2 \mathrm{z}, \quad \Delta \Theta_{\mathrm{t}}=2\left[\frac{\frac{\mathrm{dM}(\mathrm{z})}{\mathrm{dz}}(\cos \theta)^{2}}{\sin \theta} \mathrm{z}_{\mathrm{t}}-\Theta_{\mathrm{t}}(28)\right]
$$

to give the required reflection at a boundary. 


\section{CALCULATION OF TOTAL INTENSITY LOSS}

Because of reflections from the duct walls, rays traveling totally different paths could arrive at the same receiver point. To arrive at the total ray intensity loss a coherent sum of all ray arrivals was calculated. In Fig. 7 a typical receiver point is shown where five distinct ray paths connect it to the sound source. For each ray the intensity loss and phase were calculated along the ray path. The intensity loss for the combination of $n$ arrivals was then calculated from (see reference 31 )

$$
N=-10 \log \left\{\left[\sum_{j=1}^{n}\left(I_{j} / I_{0}\right)^{\frac{1}{2}} \cos \theta_{j}\right] 2+\left[\sum_{j=1}^{n}\left(I_{j} / I_{0}\right)^{\frac{1}{2}} \sin \theta_{j}\right] 2\right\} \text {. }
$$

The phase angle for each ray was calculated from

$$
\theta_{j}=2 F^{*} T_{j *}
$$

where $T^{*}$ is the nondimensional time of arrival of ray $j$ and $F^{*}$ is the nondimensional frequency parameter of the source.

The calculation of intensity using this procedure required the identification of all significant rays that traveled from the source to an array of receivers. There could be an infinite number of rays which travel to a single receiver, but the rays with sufficiently large values of $\theta_{0}$ from the source will bounce off the walls many times and can be neglected. In this study all rays which were reflected from the duct walls more than twice were not considered.

Finding all significant ray paths was a major numerical calculation problem. The difficulty appeared when trying to 


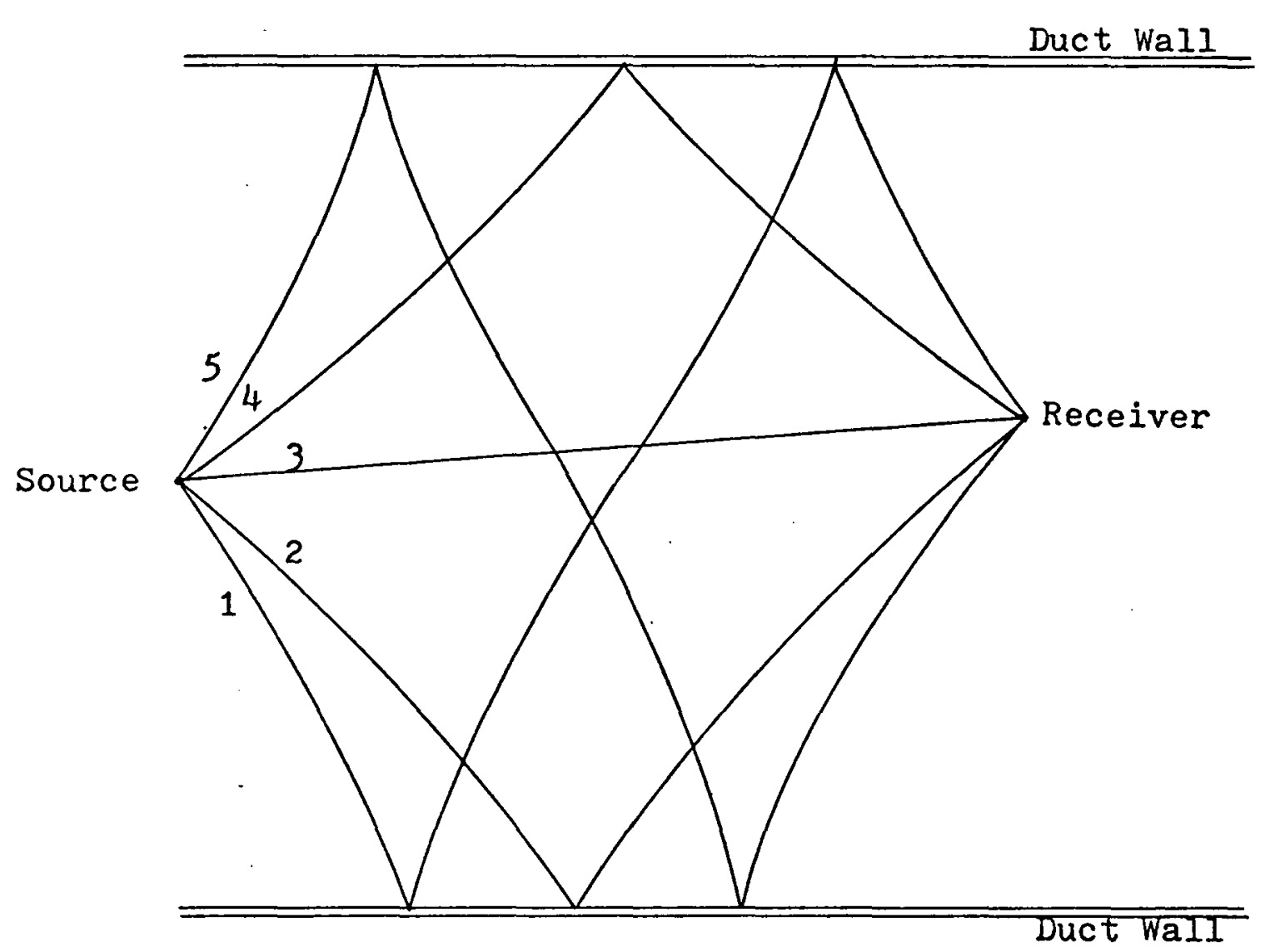

Fig. 7 A Typical Set of Ray Paths 
constrain the ray trace solution to pass through a fixed end point at some unknown time along the ray path. One possible method which was attempted involved calculating the entire ray path until it crossed a specified cross section of the duct. The height of the ray at this point was then compared to the receiver height and a proportional change in the initial ray inclination angle was made on the next trial. This method had some problems with convergence and it was also a very inefficient method. The computer time required to calculate data ruled out this approach.

An approach which proved to be much more efficient was the ray sweep-out method (32). Since the phase velocity varied only with height, it was possible to calculate each ray to its first wall reflection and from that to predict its final position relative to the receiver point. Shown in Fig. 8 is a typical ray path. The horizontal distances at which the ray crossed the receiver height and at which it intercepted the duct wall were stored. If $R$ was equal to the horizontal distance that the ray traveled before it began another cycle, then

$$
R=4\left(R_{1}+R_{2}\right)
$$

Since both the top and bottom walls of the duct are horizontal planes, the distances $R_{1}$ and $R_{2}$ completely define the ray. It was then possible to predict the horizontal distances at which the rays crossed the receiver heights in the duct without calculating the complete paths. This meant a significant 


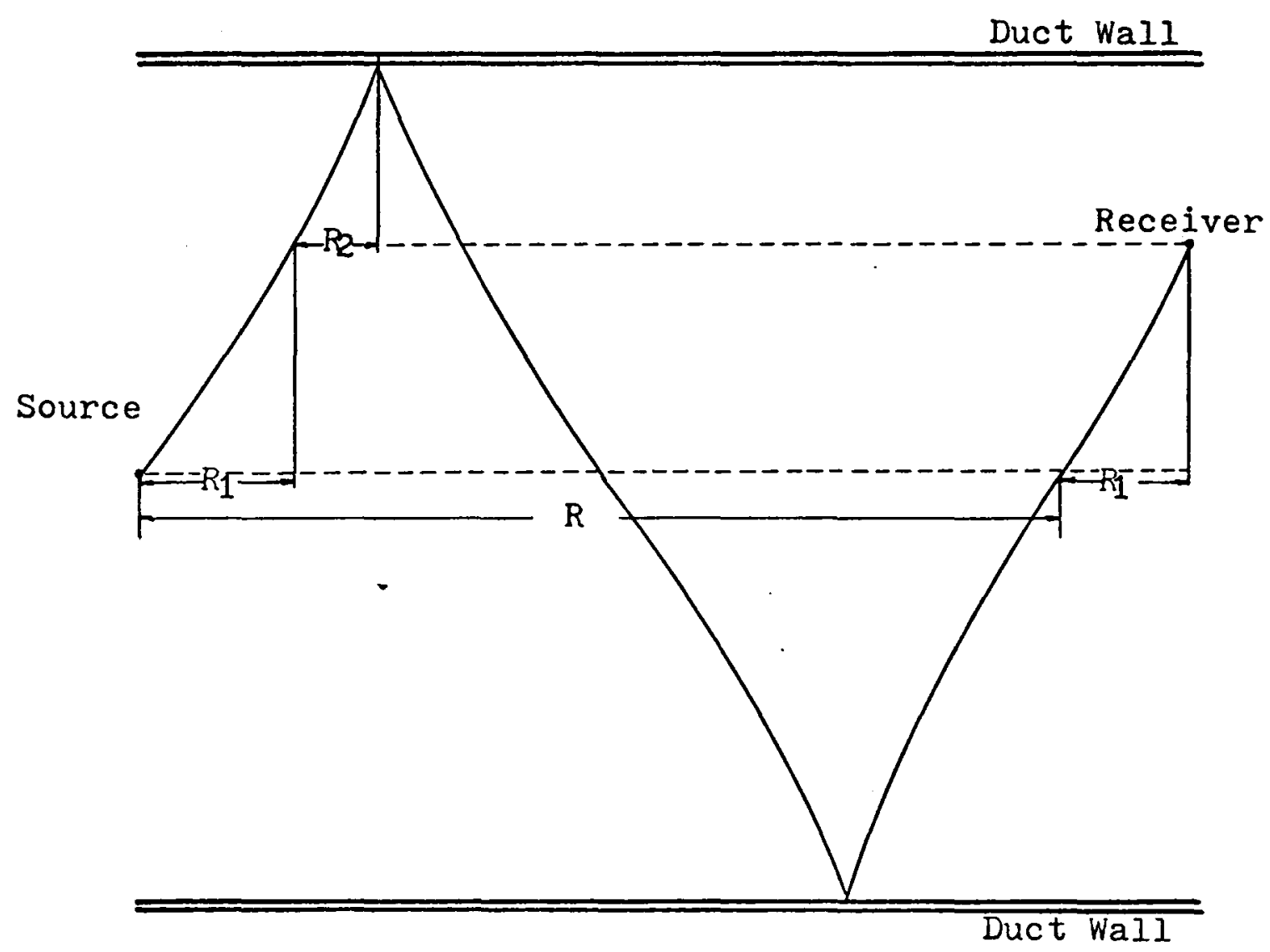

Fig. 8 Horizontal Distances Required to Define a Ray Path 
decrease in computation time. Each ray path was calculated to its first wall reflection, and then the horizontal distance at which the ray reached the receiver height nearest a specified cross section of the duct was determined from extrapolation. Depending upon the initial angle, the ray either fell beyond or fell short of the desired horizontal distance to the receiver. The ray inclination angle could then be adjusted until the correct range was found. Good results were achieved in finding the correct angle by linear interpolation between rays which bracketed the receiver position. The intensity loss profile plots were found at each cross section of the duct by performing the above calculations for a vertical linear array of one hundred receiving points across the duct. 


\section{VAIIDITY OF RAY ACOUSTICS APPROACH}

Ray acoustics when applied incorrectly can produce totally erroneous sound pressure intensity predictions. Due to the simplicity of the ray approach there is an inclination to at times rely too heavily upon its results. It is important to realize that ray acoustics at best is still only an approximate solution. For the approximation to be reasonable, the basic assumptions used in the ray theory must be valid for the particular case under study. Unfortunately the precise definitions of these assumptions and the methods for verifying them are perhaps the least developed part of the ray theory at present.

The goal must be to determine if the solution of the eikonal equation is approximately equivalent to the rigorous wave equation solution. Blokhintzev (25) derived the eikonal equation as a linear approximation to the wave equation. The validity of his derivation was dependent upon the correctness of two assumptions. First, in order to linearize the wave equation the perturbations of the medium had to be small. Second, the variation in the properties of the medium must be small over a distance of one wavelength of radiation. These conditions are meaningless, however, unless there is some procedure for making quantitative checks for their satisfaction.

One means for testing the validity of the ray approach 
involves a testing function formed from a knowledge of the medium structure and sound frequency. For the ray theory to be valid, the index of refraction $M$ must not vary appreciably in a distance equal to one wavelength of radiation. This condition can be quantitatively expressed by the inequality shown by Kerr (15) to be

$$
\frac{1}{\mathrm{k}_{\mu}} \frac{|\nabla \mu|}{\mu} \ll 1
$$

where $\mathrm{k}=2 \pi / \widehat{C}$ is the wavenumber. To make the inequality statement more definite a ratio of $10^{-2}$ or less was specified as a validity criterion.

For sound propagating in a duct with a sheared flow, the index of refraction is equal to the ratio of the speed of sound and the phase velocity of the wave. Using the expression derived earlier for the phase velocity, this ratio can be written as

$$
\mu=\frac{c}{c+v(z) \cos \theta}
$$

where $\theta$ is the wave normal angle. The index can be expressed in nondimensional terms by dividing through each term by the speed of sound to arrive at the following result:

$$
\mu=\frac{1}{I+M(z) \cos \theta}
$$

where $M(z)$ is the flow Mach number. The magnitude of the gradient of the index of refraction will therefore be just the derivative with respect to $z$ which can be written as

$$
|\nabla \mu|=\frac{\frac{\mathrm{d} M(z)}{\mathrm{d} z} \cos \theta}{(1+M(z) \cos \theta)^{2}}
$$


With this result equation 25 can be re-written in terms of the variables involved in the duct problem as

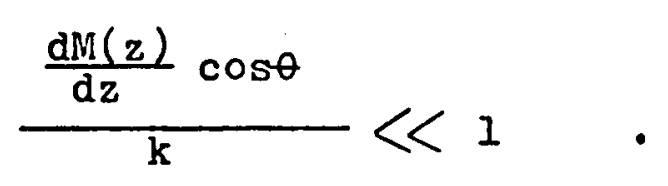

This simple relationship sums up the factors which influence the ray solution validity. Also this condition can be checked before the ray paths are actually traced from a knowledge of the structure of the medium and the frequency of the source.

From equation 29 certain conclusions can be formulated concerning the factors affecting validity. First, the quotient grows larger for increasing flow gradients. Higher flow gradients cause increased refraction of the ray paths. Ray theory predicts that all sound energy will flow along the ray paths, but in reality some energy must flow in directions other than the ray paths. In highly sheared flows the ray theory's failure to account for energy diffusion becomes much more critical to the results. Secondly, equation 29 is more likely to be satisfied for rays with large wave normal angles. This result is evident from the definition of the phase velocity. The phase velocity equals the speed of sound plus the dot product of the flow velocity vector in the wave normal direction. For rays directed with near normal incidence to the duct walls the angles between the flow direction and the wave normals are nearly right angles. Therefore the change in phase velocity due to the flow is small as the ray propagates across the duct. This results in only a small change 
in the index of refraction and very little bending of the rays. For rays with low angles of incidence, this dot product increases in magnitude and the phase velocity varies a greater amount due to the flow velocity gradients. For rays with near grazing angles through the shear layers, the theory predicts greater bending of the rays and higher spreading losses. Thirdly, the inequality implies that acoustic ray formation will be more valid for large wavenumbers and thus high frequencies.

For this study, the ray which entered the shear layer at the lowest angle of incidence at the specified cross section of the duct under study was examined. The flow gradients in the shear layers were then approximated as a constant average value for use in equation 29. It was then possible to solve for the wavenumber which gave the lowest frequency acceptable. For the range of centerline Mach numbers and boundary-layer thicknesses considered, Table 1 gives the average flow gradients in the various shear layers and the maximum wavelengths for valid results with downstream propagation.

For upstream propagation of sound the rays encounter turning points in the shear layers because of the refraction of the rays towards the duct center. The wave normal angle equals zero at these points and its cosine approaches the maximum value of unity. Equation 29 can then be written as

$$
\frac{\frac{\mathrm{d} M(z)}{\mathrm{dz}}}{\mathrm{k}}<1
$$


Table 1

Maximum Wavelengths for Valid Application of Ray Acoustics

$\begin{array}{ccccc}\begin{array}{c}\text { Centerline } \\ \text { Mach No.. M Ml }\end{array} & \begin{array}{c}\text { Boundary-laver } \\ \text { Thickness }(\mathrm{d} / \mathrm{H})\end{array} & \begin{array}{c}\text { Average Flow } \\ \text { Gradient }(\mathrm{M} / \mathrm{H})\end{array} & \begin{array}{c}\text { Upstream } \\ (S \max / \mathrm{H})\end{array} & \begin{array}{c}\text { Dowmstream } \\ (\mathrm{Smax} / \mathrm{H})\end{array} \\ 0.3 & 0.3 & 1.07 & 0.059 & 0.092 \\ 0.5 & 0.1 & 6.05 & 0.010 & 0.013 \\ 0.5 & 0.2 & 2.76 & 0.022 & 0.034 \\ 0.5 & 0.3 & 1.78 & 0.035 & 0.050 \\ 0.5 & 0.4 & 1.31 & 0.049 & 0.074 \\ 0.7 & 0.3 & 2.49 & 0.025 & 0.044\end{array}$


With this equation it was then possible to determine the minimum frequencies allowable for valid upstream results. These values were calculated and tabulated in Table 1, also. The frequencies determined by use of equation 25 represent the lower limits for valid application of ray acoustics. Although these limits are necessary conditions, they do not constitute sufficient conditions. An additional requirement which must be satisfied is that the fractional change in the spacing between neighboring rays must be small in a wavelength of radiation. A ray pattern therefore leads to questionable results in regions where rays either diverge or converge rapidly, and especially where neighboring rays cross to form foci or caustics. This second condition can not be determined in advance but can be determined after the ray pattern has actually been traced. For the flow duct problem it was found that the formation of caustic lines for the upstream case made intensity predictions impossible with simple ray theory. 


\section{DISCUSSION OF RESULTS}

The solutions to the ray trace equations and the spreading loss equations were evaluated numerically through the use of a Runge-Kutta integration routine. The output of the computer program was put in the form of acoustic ray plots and intensity loss plots for specified cross sections of the duct. For downstream propagation, six cases were considered in an effort to show the effects of boundary-layer thickness, centerline Mach number, and frequency on intensity 1oss. For $M_{C I}=0.5$ the effects of varying the flow boundarylayer thickness were considered for shear layers equal to 10 percent, 20 percent, 30 percent, and 40 percent of the duct height. The effects of centerline Mach number on the sound field were considered for $\mathrm{M}_{\mathrm{Cl}}=0.3,0.5$, and 0.7 with a fixed boundary-layer equal to 30 percent of the duct height. Each intensity loss profile was calculated at a distance equal to one duct height from the sound source for different frequency parameters $\left(f^{*}=10,20\right)$. (Note: The frequency parameter $f^{*}$ is equal to the number of sound wavelengths which will fit across the height of the duct.) For the upstream propagation, it was not possible to calculate intensity plots with the present theory so only ray plots were calculated.

\section{A. Ray Plots}

The ray plots shown in Figs. 9, 10, and 11 for upstream 
propagation are characterized by the presence of caustics. From a purely geometrical point of view, the location of a caustic can be identified as the envelope formed by a family of rays. Ray methods can not be used to calculate the sound field on or near these lines, because the ray-bundle area shrinks to zero. Since the intensity along a ray is inversely proportional to the area of the ray-bundle, an infinite intensity would be predicted along each caustic line. In reality, the intensity near the location of a caustic will not be infinite, but the region will be a region of high energy concéntration.

To develop a valid theory in the region of a caustic, it is necessary to utilize a higher-order approximation to the general wave equation. As previously noted, the eikonal equation which forms the basis for ray acoustics is only a linearized form of the general wave equation. In the vicinity of a caustic the nonlinear terms in the wave equation can not be neglected. A nonlinear solution to the wave equation, however, was beyond the scope of this paper. Existing theories for the solution of sound fields near a caustic can be found in references 16 and 33 .

Although it was not possible to calculate intensity losses for upstream propagation, the ray plots provide a good indication of where the acoustic energy should be concentrated. The highest concentration of energy should occur in the shear layers near the caustics. In the central part of the duct, 


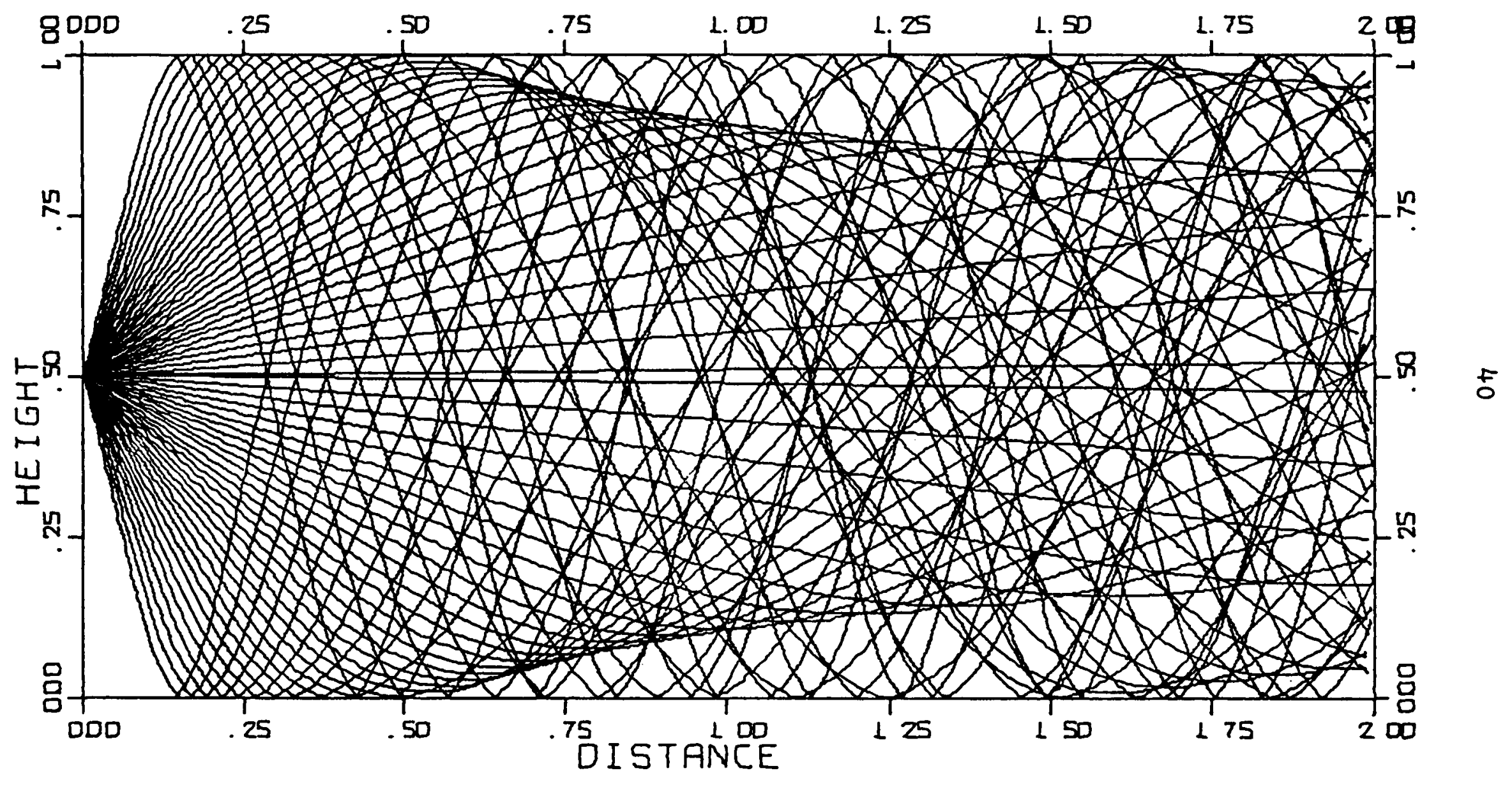

FIG. 9 RAY DIAGRAM FOR UPSTREAM PROPAGATION, CLM $=03, B L T=03$. 


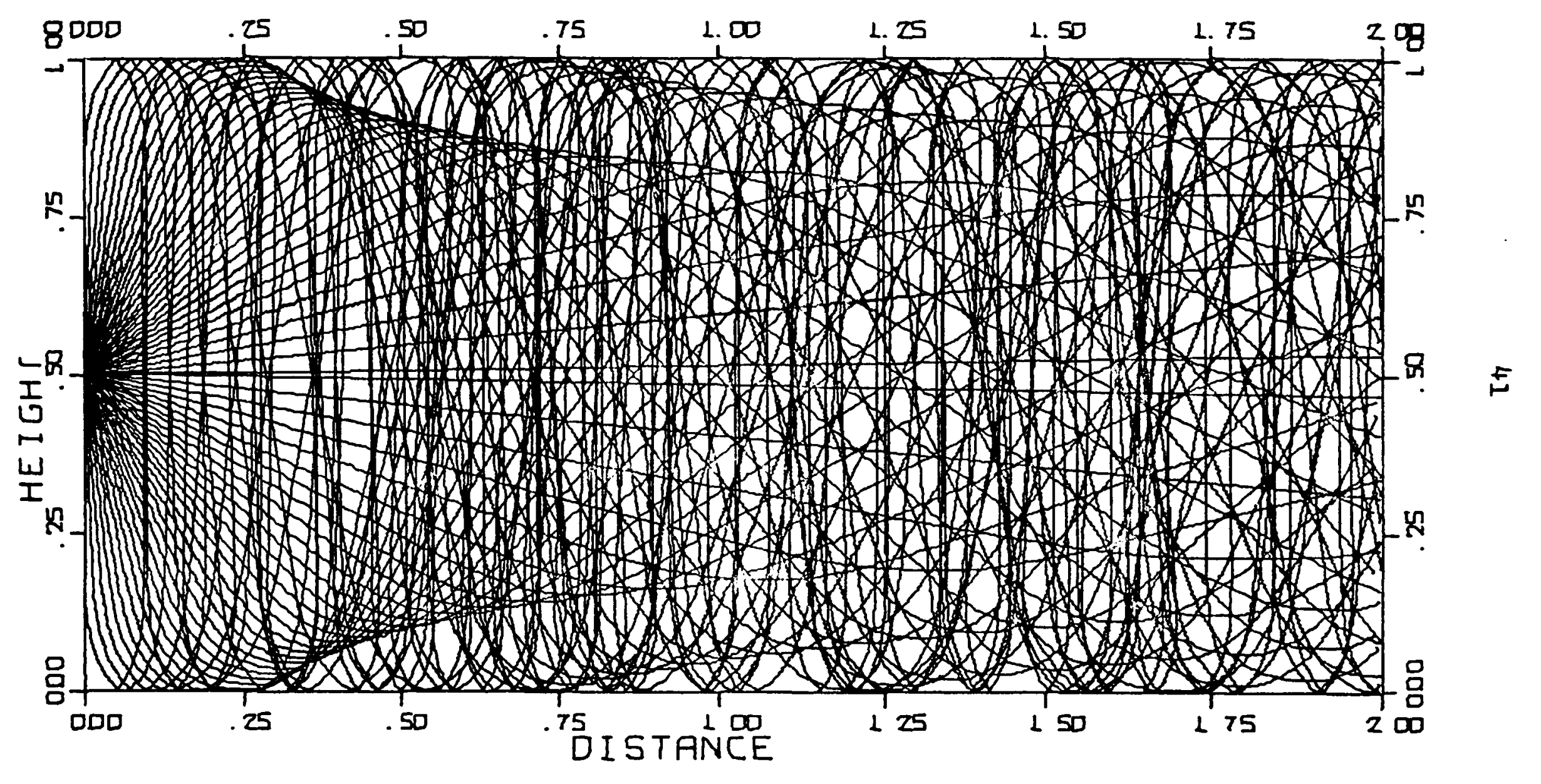

FIG. 10 RAY DIAGRAM FOR UPSTREAM PROPAGATION, CLM $=0.5, B L T=0.3$. 


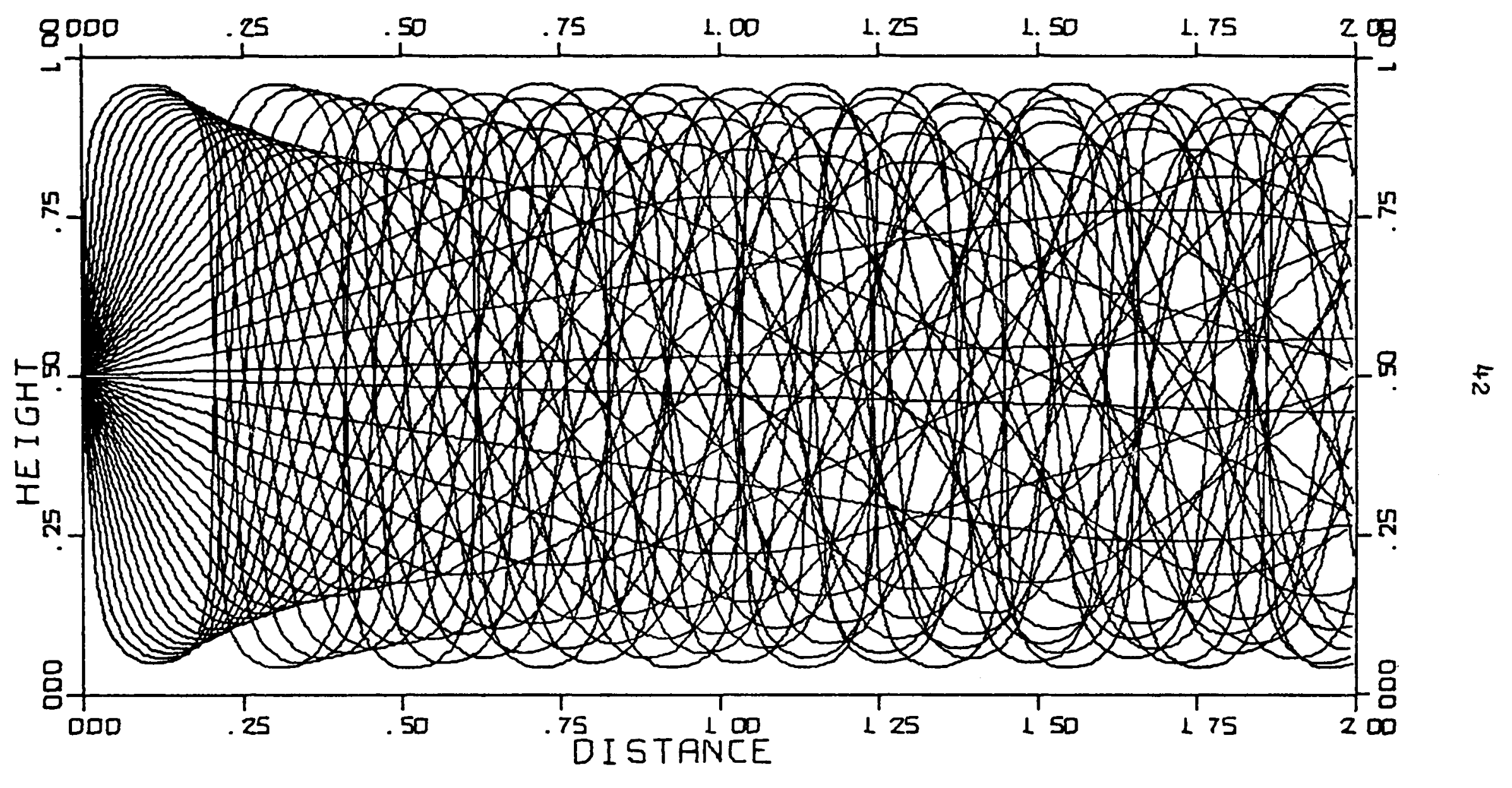

FIG. 11 RAY DIAGRAM FOR UPSTREAM PROPAGATION, CLM $=07, B L T=03$. 
the intensity should oscillate due to the interference between the reflected and turned rays. In the regions where there are no rays adjacent to the duct walls, such as in Fig. 9. ray theory predicts a shadow zone with complete silence. Higher order solutions to the sound field near a caustic have shown, however, that in such regions the sound field decreases monotonically with distance from the maximum near the caustic.

Each of the ray plots shown for five downstream cases under study are all very similar in appearance. Unlike the upstream ray plots, the bending of the rays was not as pronounced in the shear layers. In each plot the rays are straight in the constant velocity region of the duct and then bend slightly towards the duct wall in the shear layer region. Less bending was observed for the downstream case, because the flow gradients did not contribute to variations in the index of refraction to the degree present in upstream propagation. This was because the index of refraction $\mu$ is related to both the flow Mach number $M(z)$ and the cosine of the angle $\theta$ between the wave normal and the flow velocity direction by the relationship

$$
\mu=\frac{1}{1+M(z) \cos \theta}
$$

For the higher downstream angles of incidence the component of flow velocity in the direction of propagation was very small. Thus variations in the flow velocity had small effects on the wave phase velocity. For upstream propagation the rays were bent in the direction of decreasing wave normal angles 


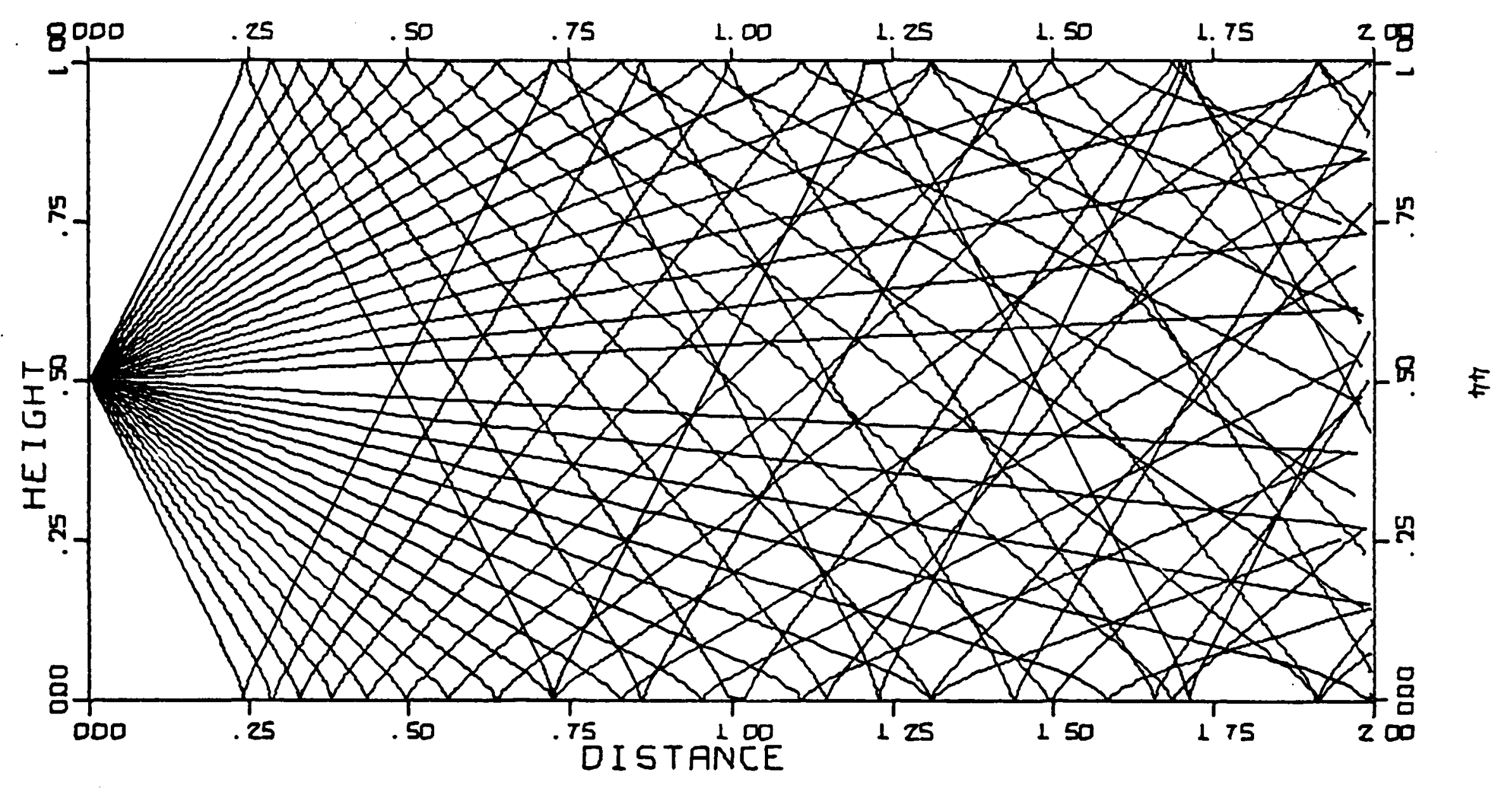

FIG. 12 RAY DIAGRAM FOR DDWNSTREAM PROPAGAT ION, CLM. D.5, BLT.01. 


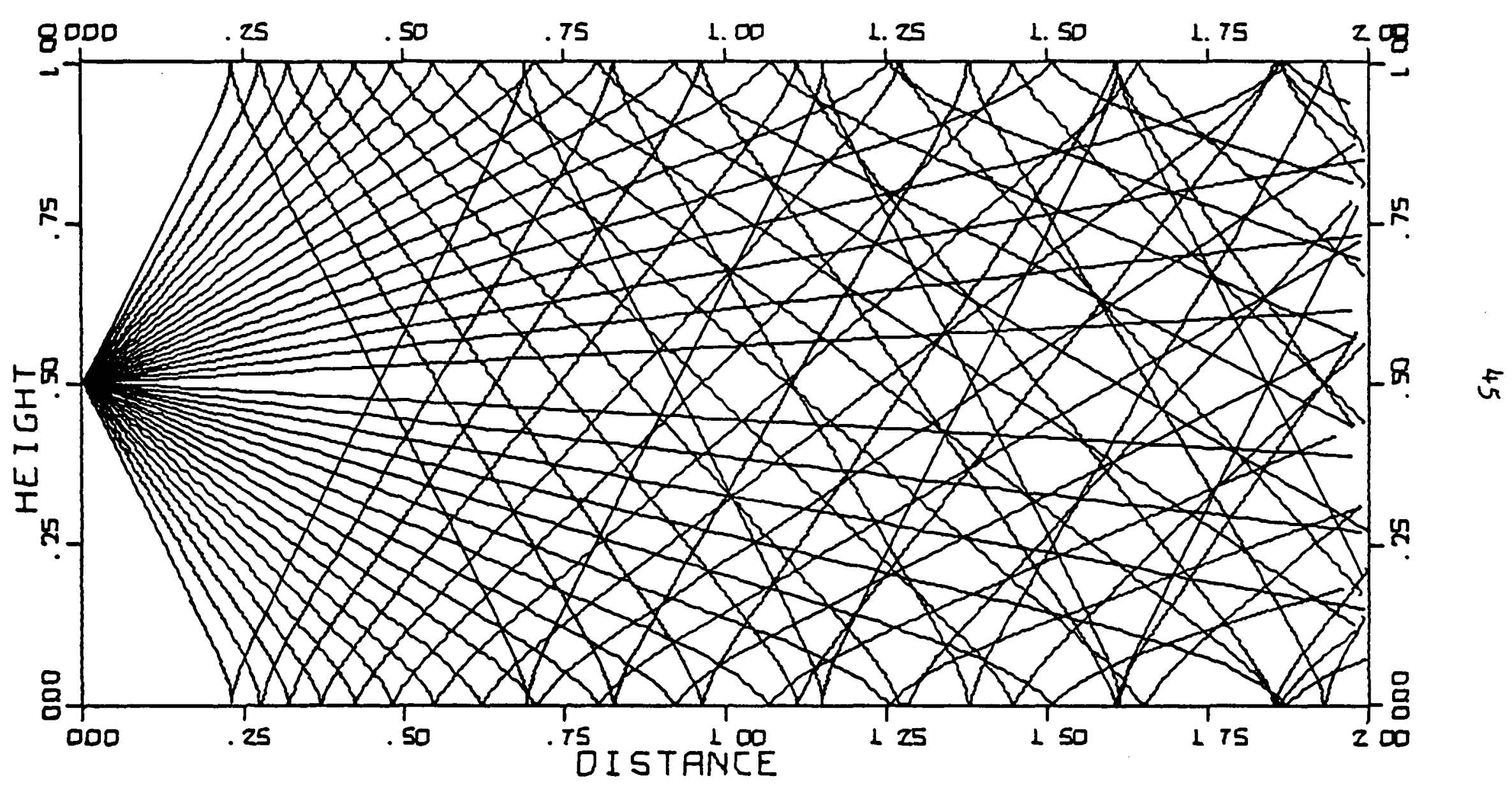

FIG. 13 RAY OIAGRAM FOR DOWNSTREAM PROPAGATION, CLM.DS, BLT-02. 


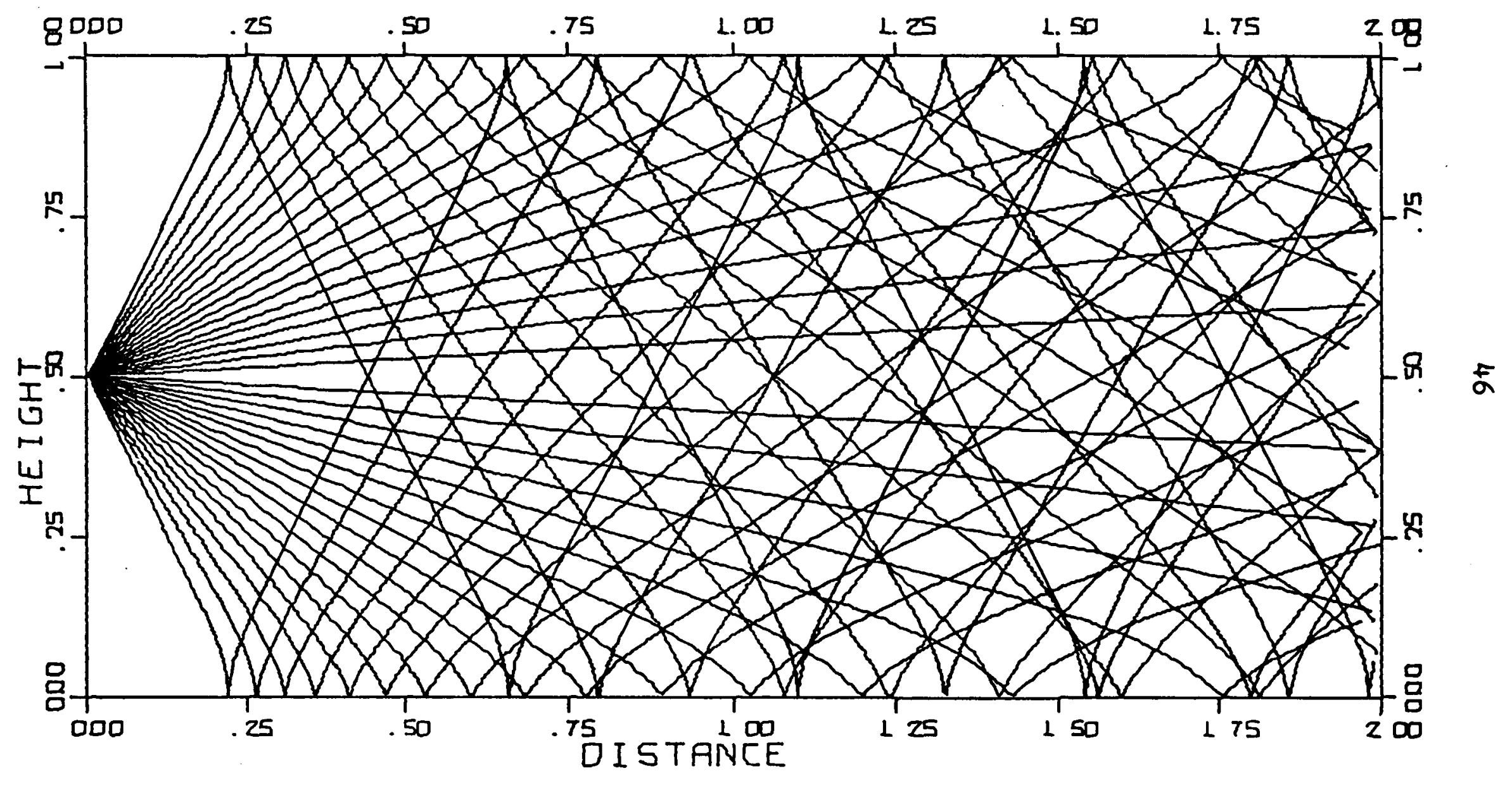

F IG. 14 RAY DIAGRAM FOR DOWNSTREAM PROPAGAT ION, CLM.0.5, BL T=0.3. 


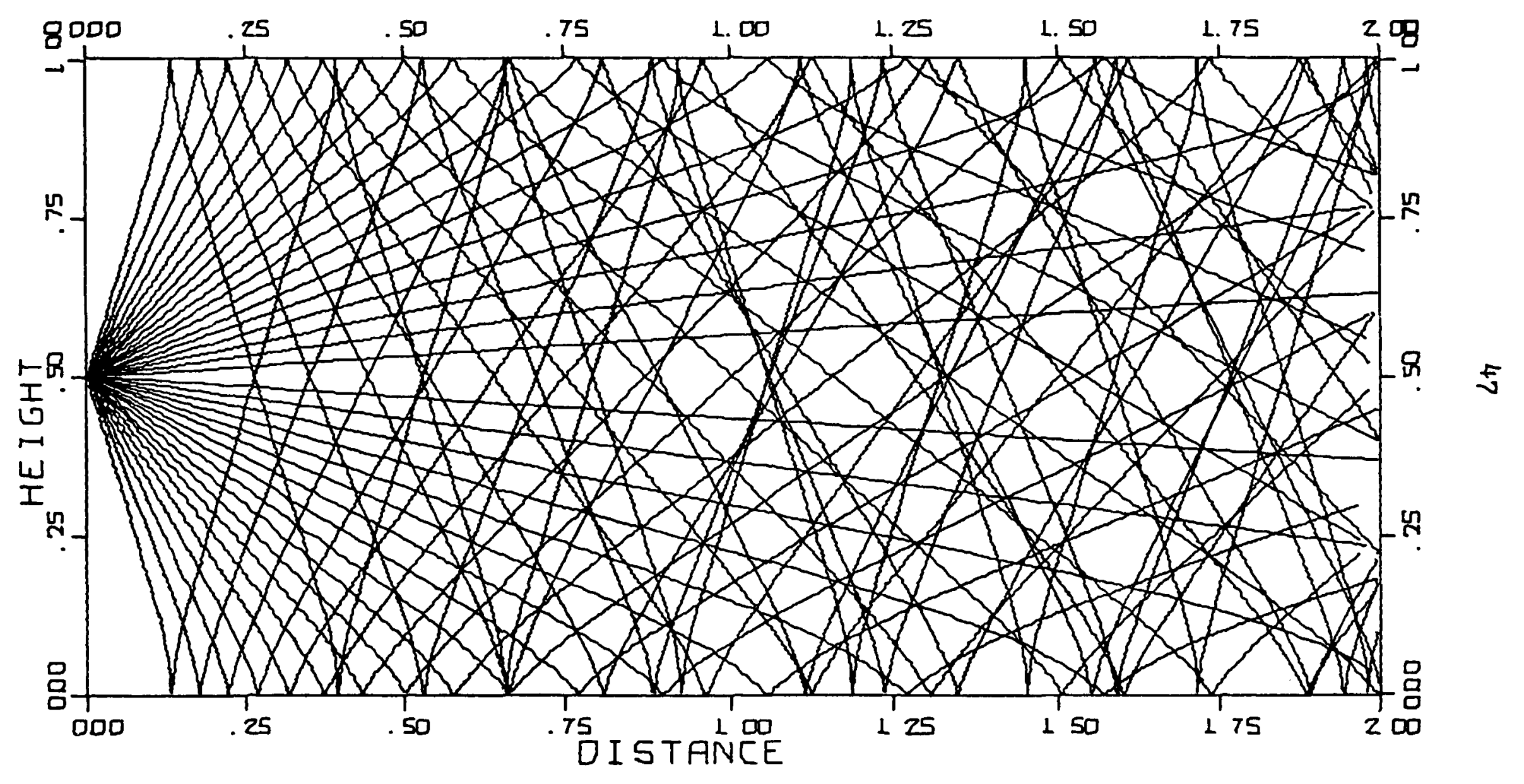

FIG. 15 RAY DIAGRAM FOR DDWNSTREAM PROPAGATION, CLM=0.3, BL T=3. 


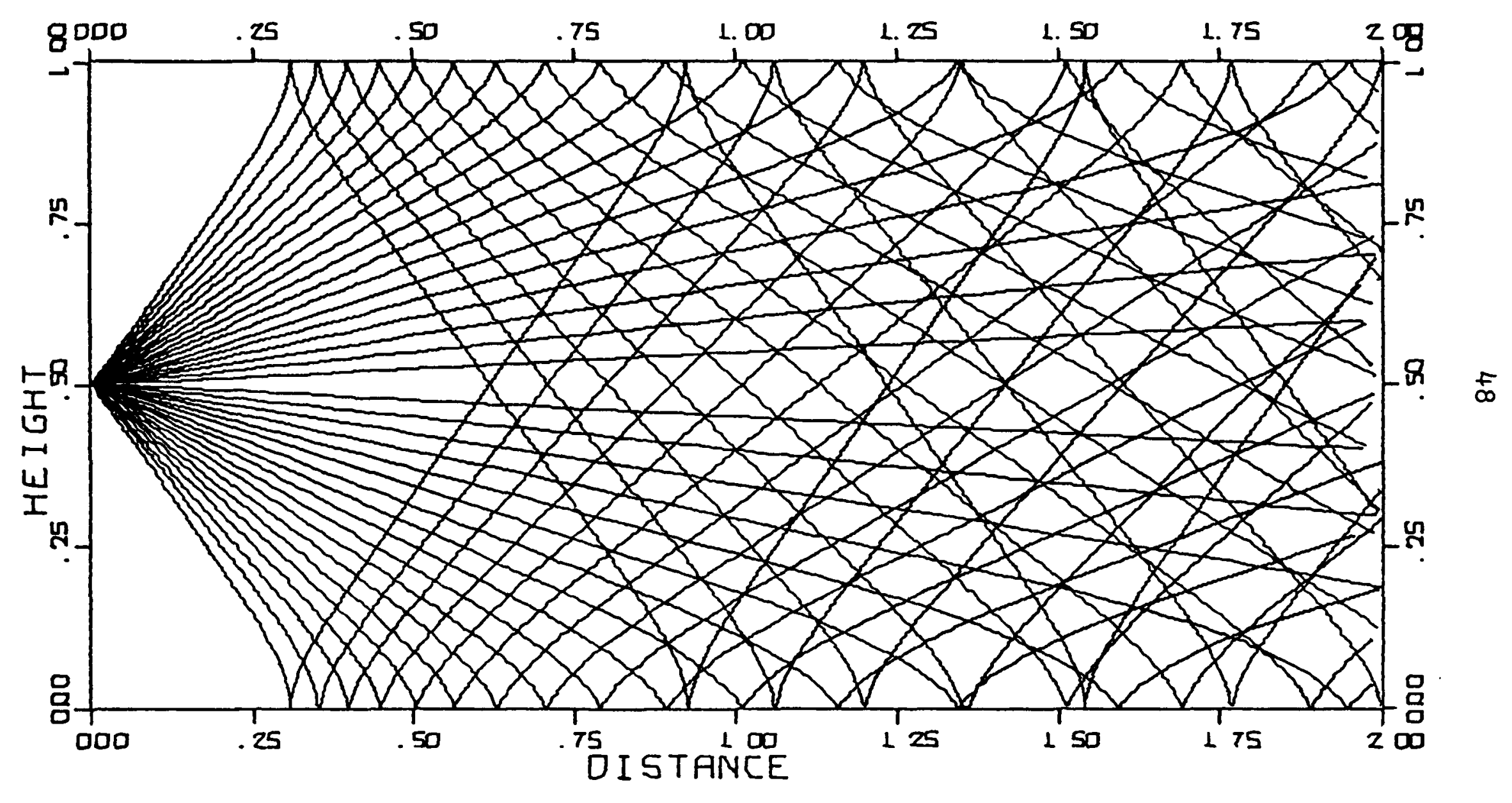

FIG. 16 RAY DIAGRAM FOR DOWNSTREAM PROPAGAT ION, CLMJJ, BLT.J.3. 
to the point where some rays even reached turning points. For these low angles of incidence the contribution of the flow velocity component to the phase velocity was maximum. Flow gradients caused greater changes in the phase velocity and thus caused greater refraction of the rays.

The phase velocity for the downstream rays was found to be greater than the phase velocity for the upstream rays. This result can be contributed to the component of flow convection in the direction of wave propagation. Phase velocity was defined in Chapter IV to be

$$
\overline{u_{n}}=(c+v(z) \cos \theta) \bar{n}
$$

where $V(z) \cos \theta$ was the convection term. For downstream propagation the phase velocity was always greater than the speed of sound, but for upstream propagation the flow velocity $\bar{V}(z)$ was in the negative direction causing the wave to travel slower than the normal speed of sound.

The ray velocity $\overline{u_{r}}$ defined earlier to be

$$
\bar{u}_{r}=c \bar{n}+\bar{v}(z)
$$

was also greatest for downstream propagation. The ray velocity has a significant effect on the distribution of energy in the duct, because energy flow is defined as the product of intensity and ray velocity. Therefore the total energy which flows through a given point in an instant of time is a function of both the intensity of the ray and the speed of energy transport along the ray. 


\section{B. Intensity Loss Plots}

For the intensity loss plots shown in Figs. 17 through 28, the ray sweep-out method was used to calculate the sound field at 100 receivers across the duct. The ray phase relationships were determined for the direct rays and the first and second set of reflected rays, and a coherent sum of the ray intensity losses was made. The "Lloyd mirror effect", caused by interference of the direct and reflected arrivals showed up clearly by the fluctuating intensity profiles. The plots are in terms of intensity loss as defined in Chapter $V$. Therefore, peaks correspond to regions of lower intensity, and valleys are regions of higher intensity.

Changing the frequency of the sound source had the effect of changing the phase relationship of the five rays which were combined at each receiver. In comparing the outputs for $F^{*}=10$ and the ones for $F^{*}=20$, the higher frequency parameter resulted in a greater number of intensity fluctuations. There appeared to be standing wave patterns across the duct which corresponded to the normal mode pattern of each frequency. For single frequency sound in a duct such fluctuations can be measured in practice at the normal mode frequencies.

The effect of changing the boundary-layer thickness for $M_{c l}=0.5$ was reflected in a decrease of intensity in the central part of the duct for the thicker boundary-layers and $f^{*}=10$. The effect of increasing the centerline Mach number 
was to decrease the overall intensity loss in the duct somewhat. This last result was due to the increased convection for the higher flow Mach numbers. As was expected the refraction effects for downstream propagation are very small, and changes in the boundary-layer thickness did not have a significant effect on the intensity loss profile. The major effect of the flow resulted from phase shifts between the rays that caused peaks on the plots to shift for different flows. 


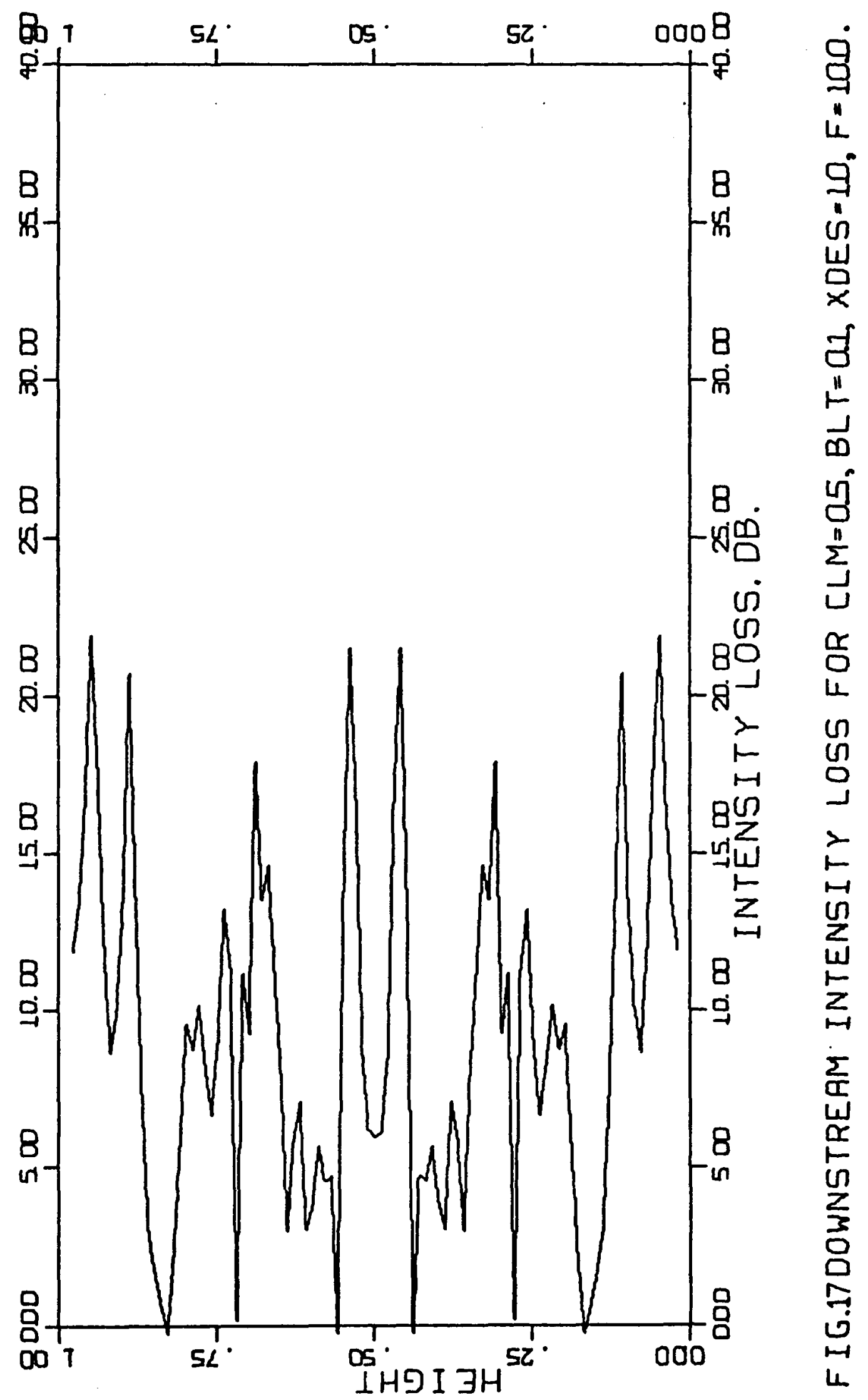




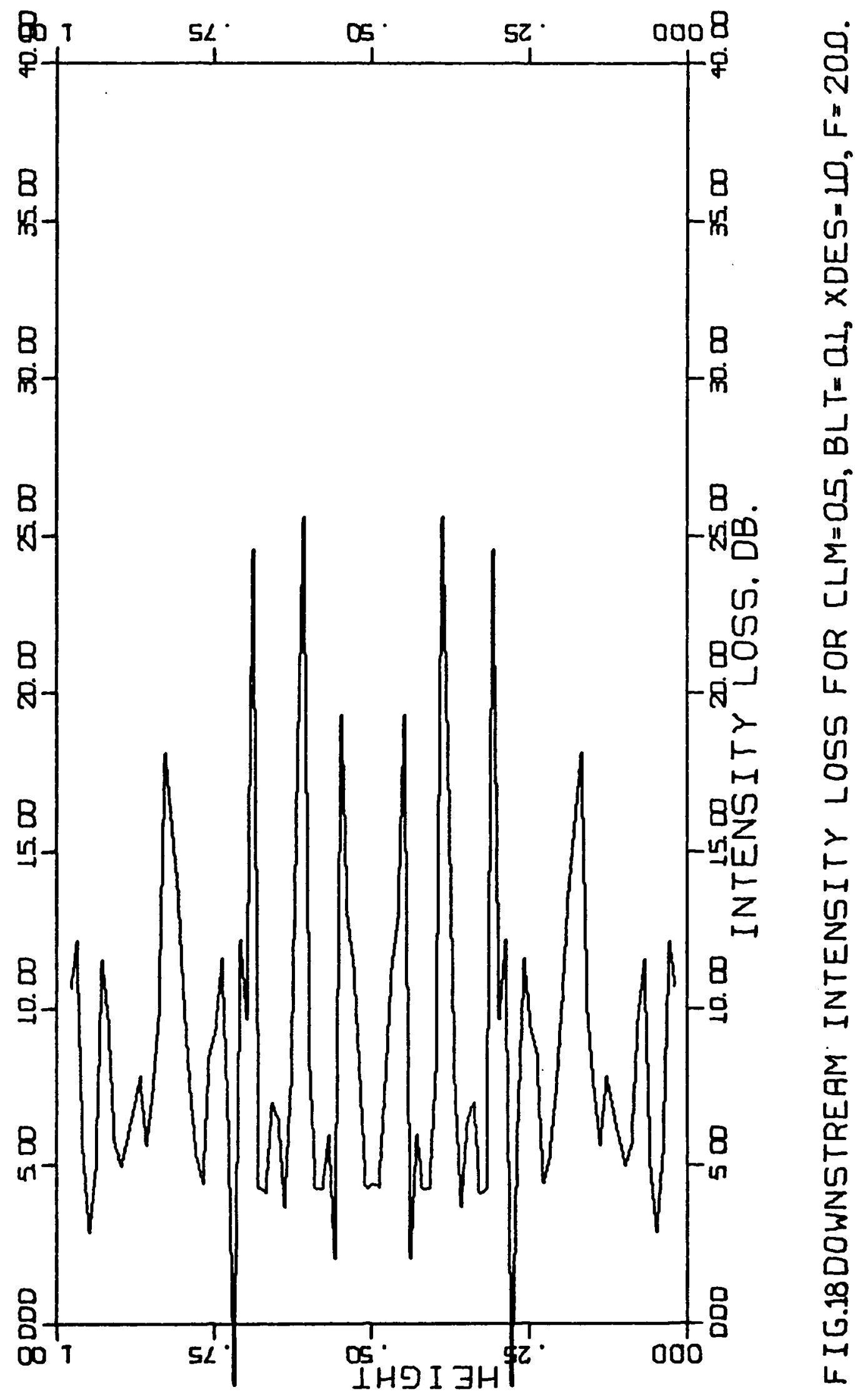




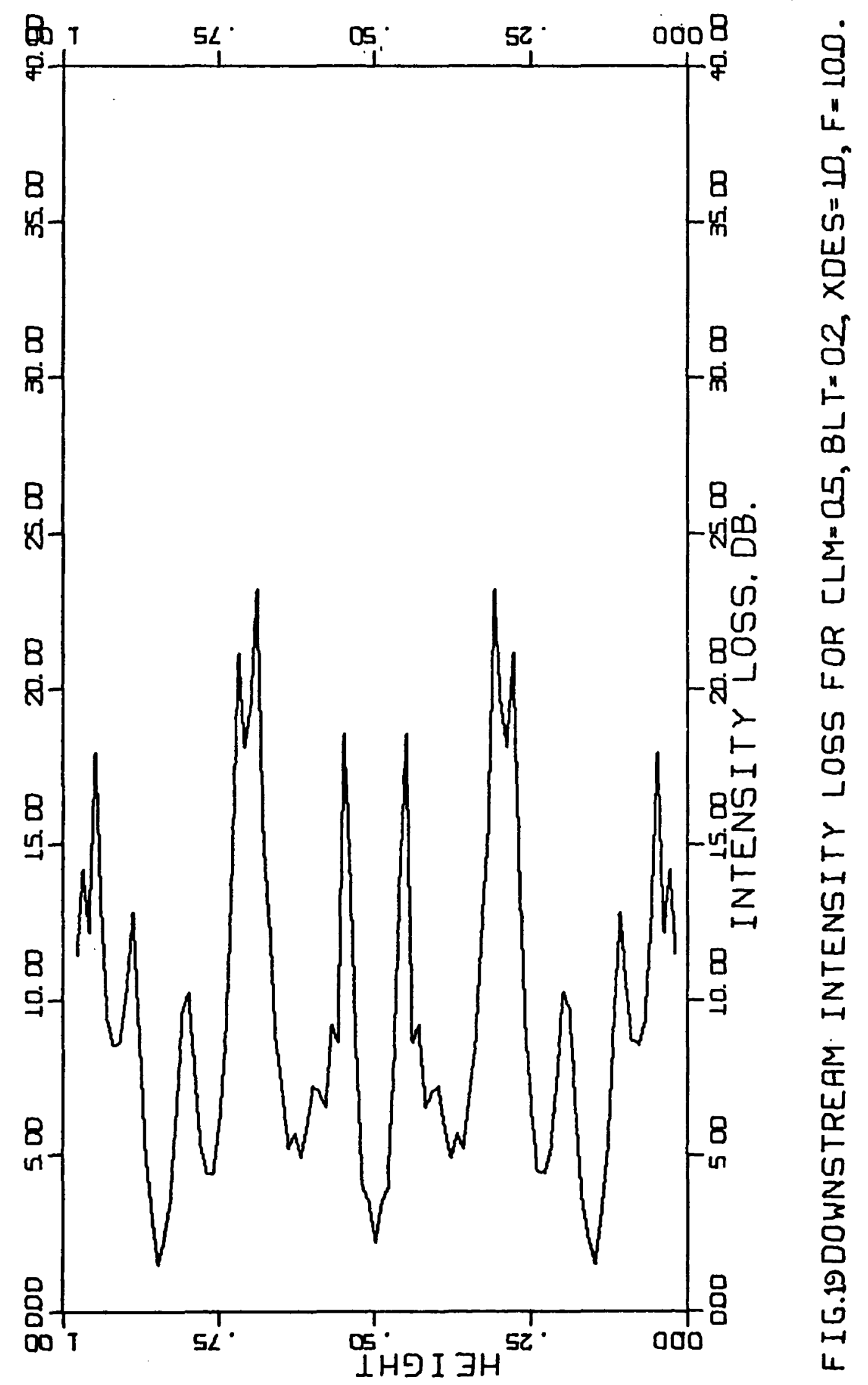




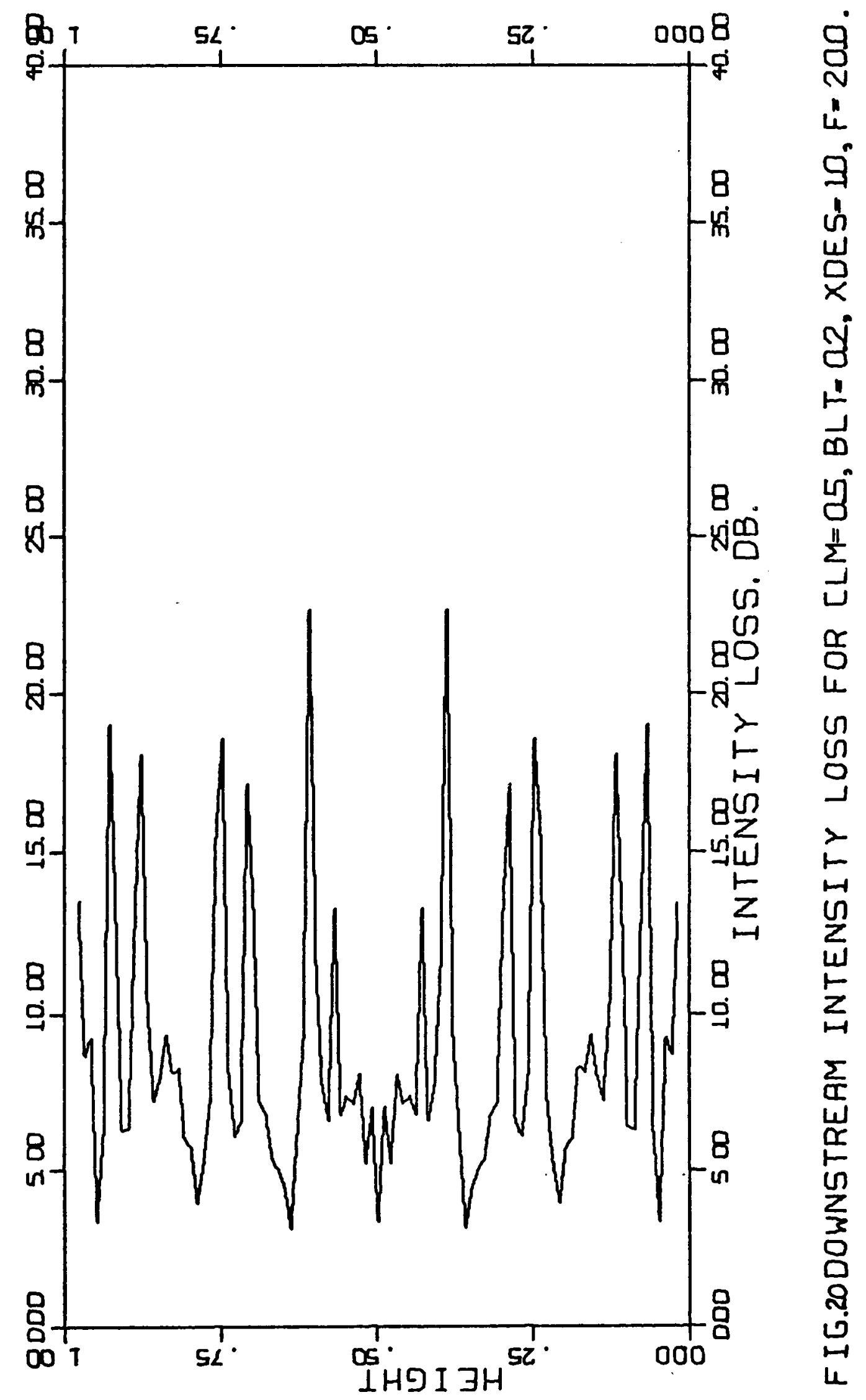




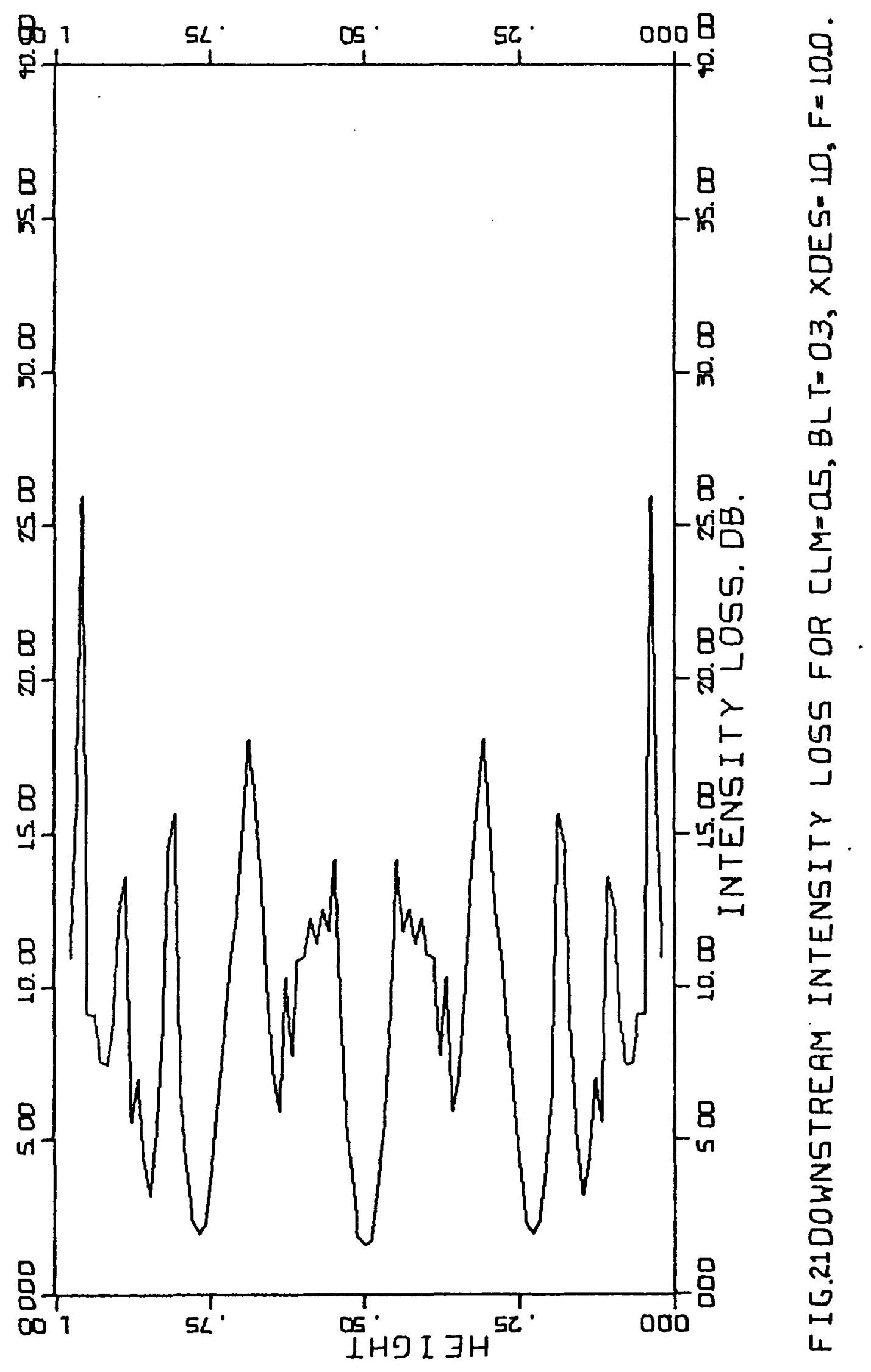




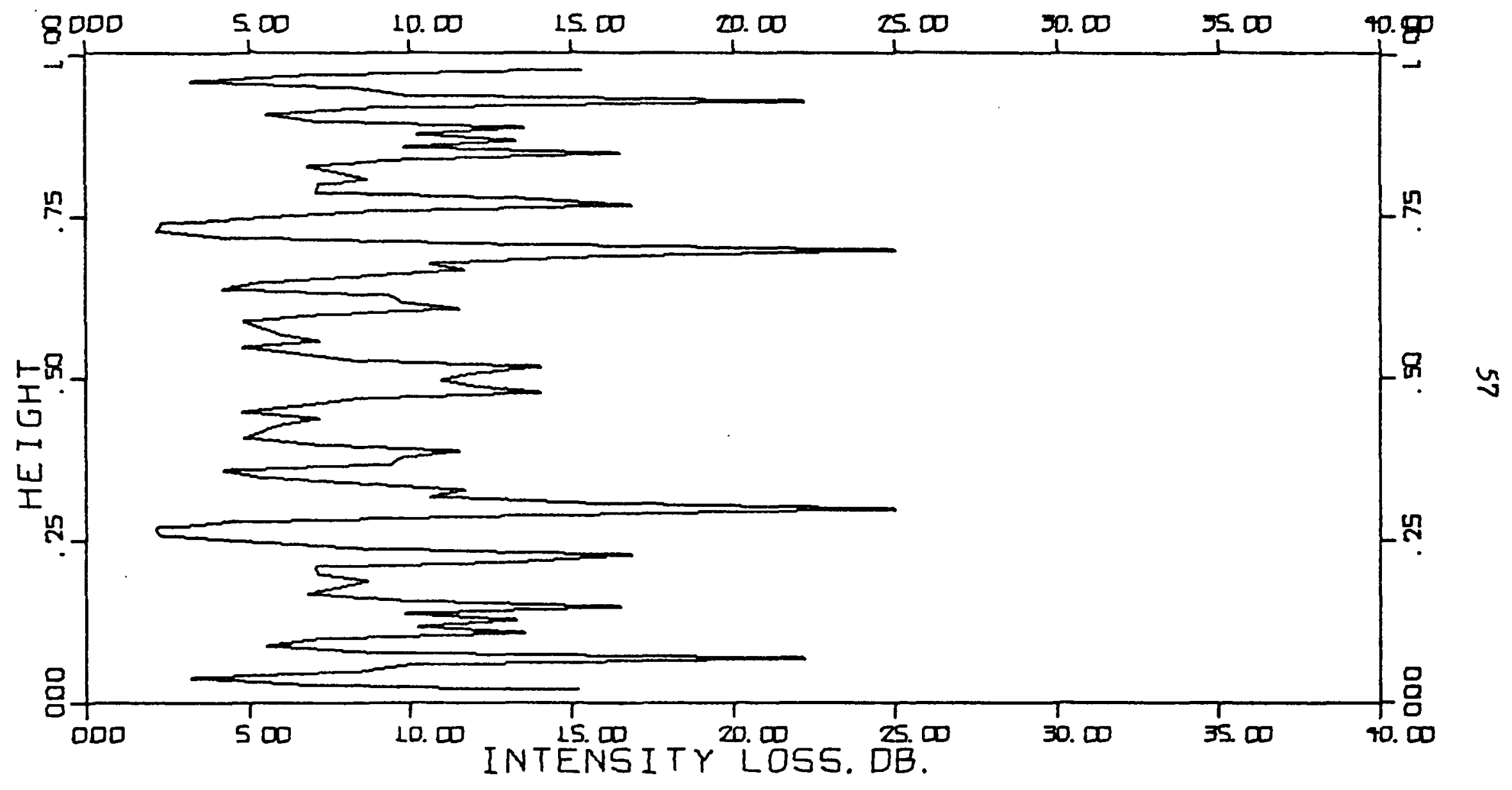

FIG.22DOWNSTRERM INTENSITY LOSS FOR CLM=05, BLT=03,XDES=10,F=20D . 


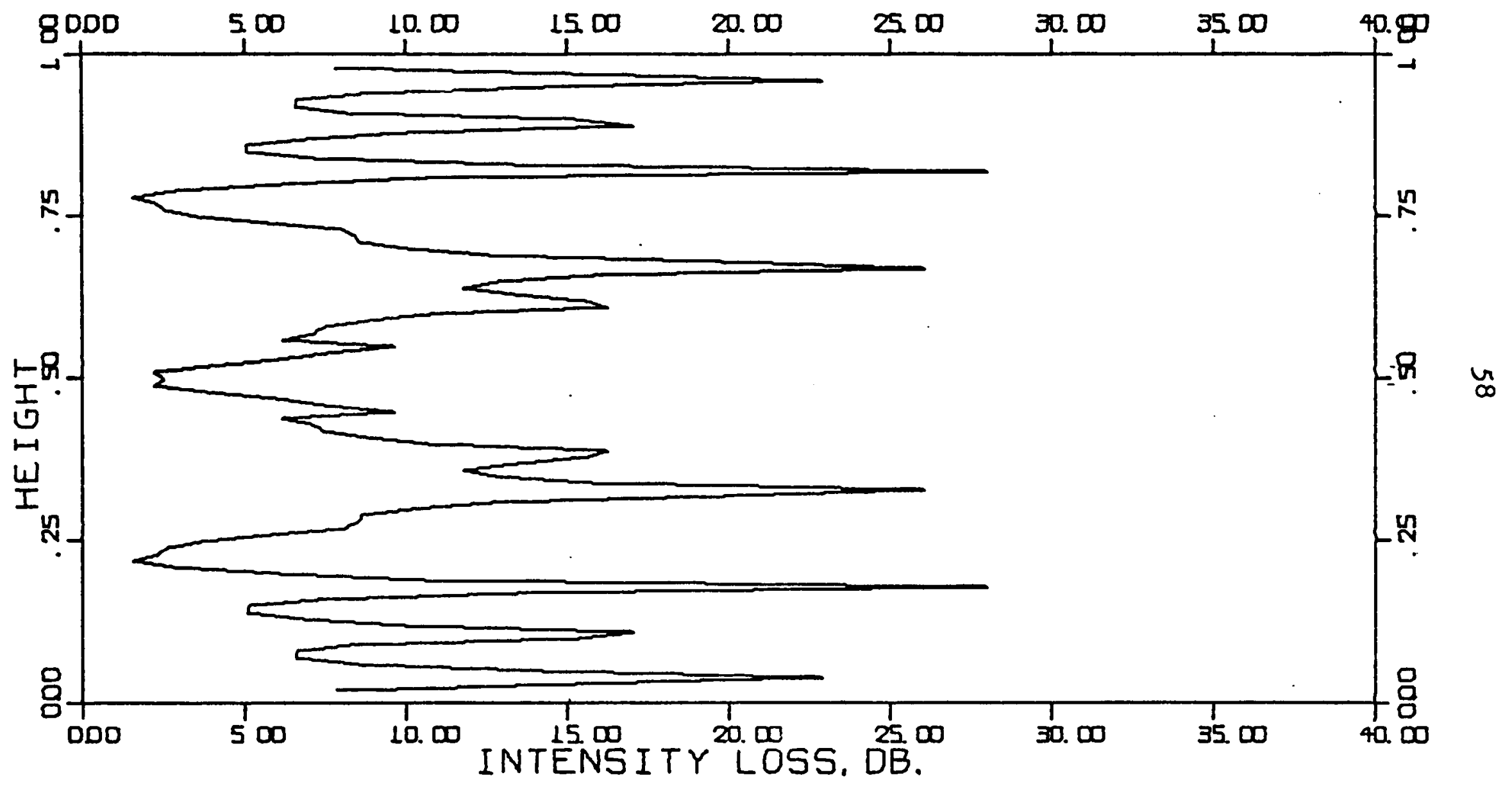

FIG.23DOWNSTREAM INTENSITY LOSS FOR CLM=05, BLT=0.4, XDES=10, $F=100$. 


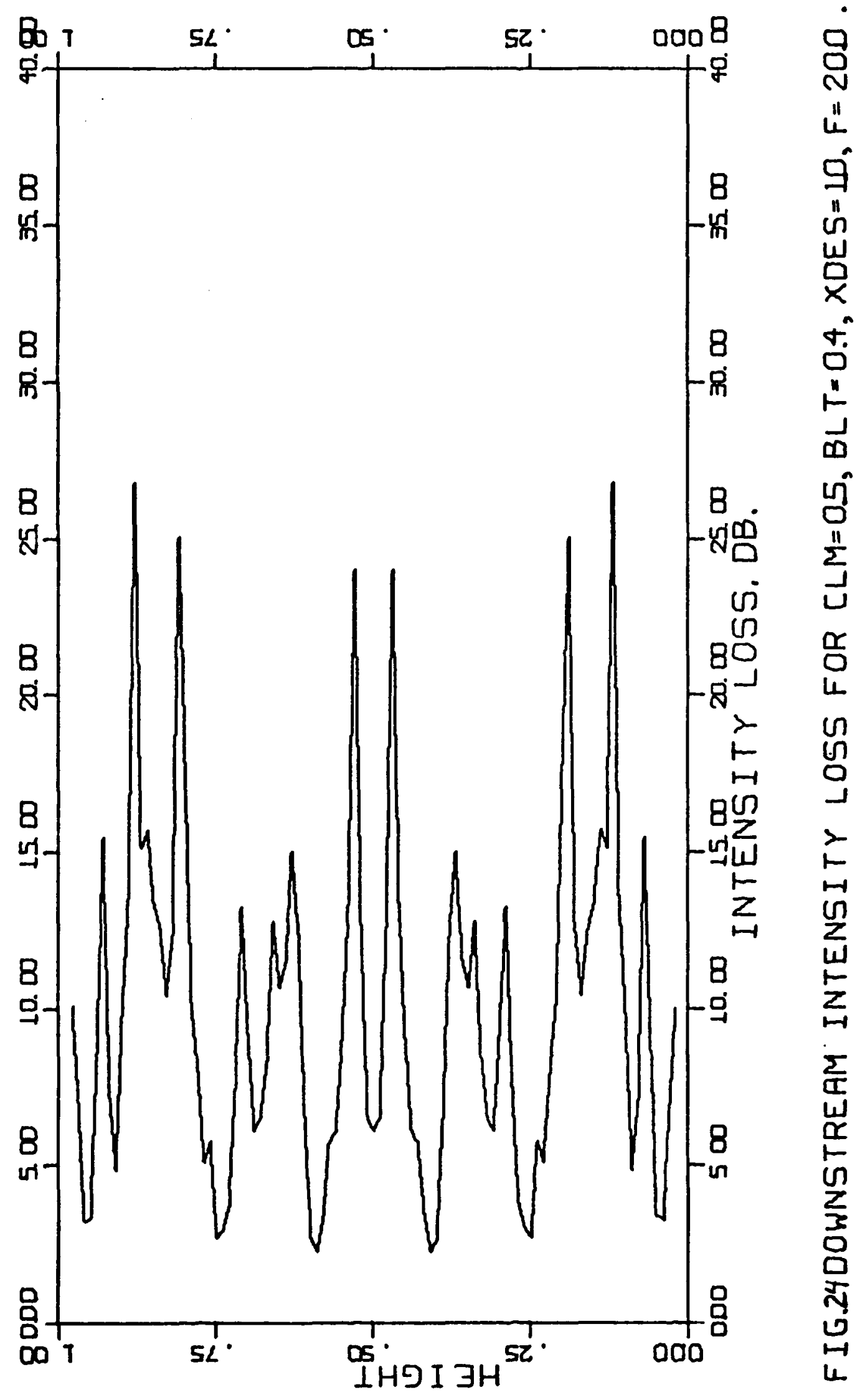




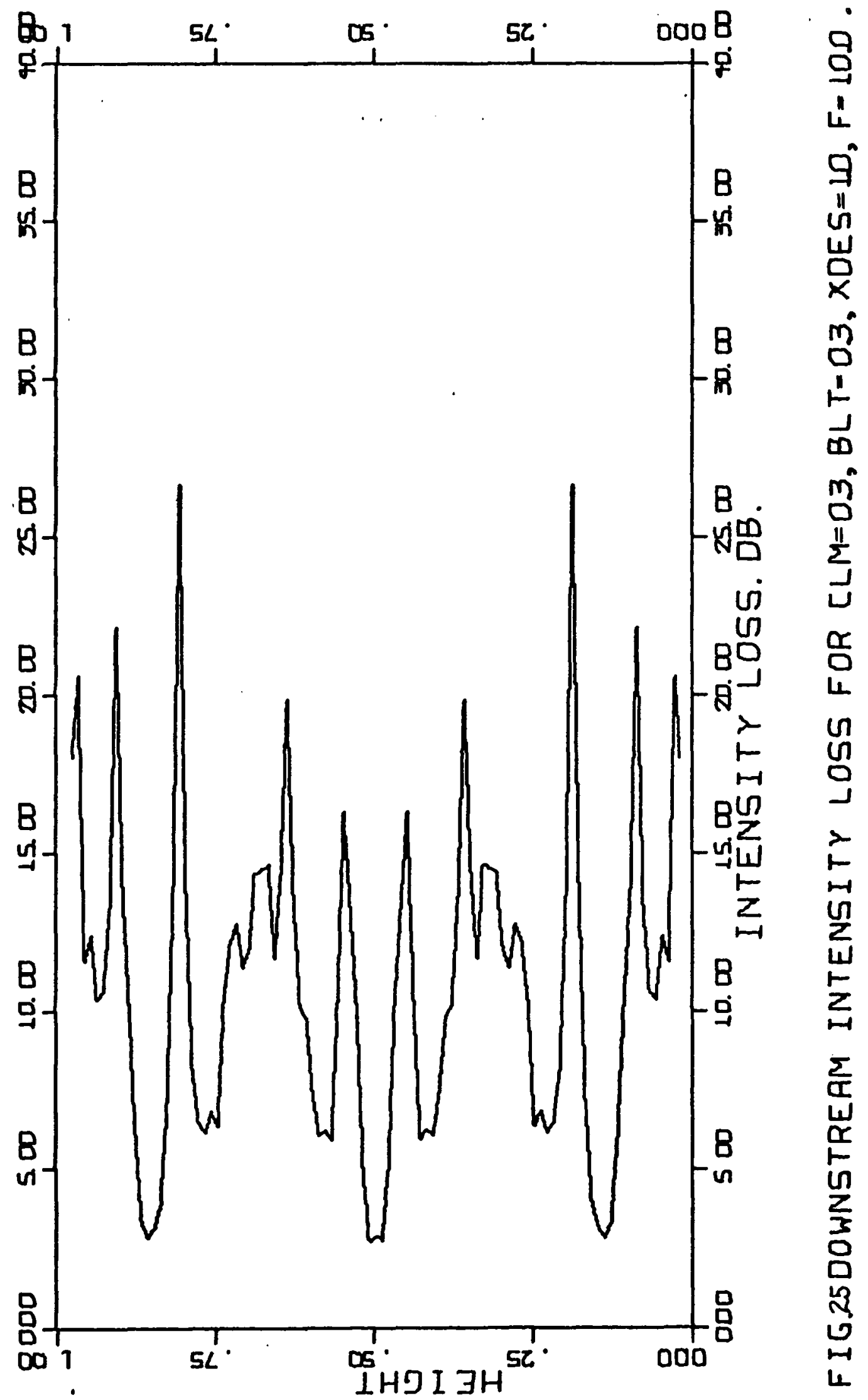


61

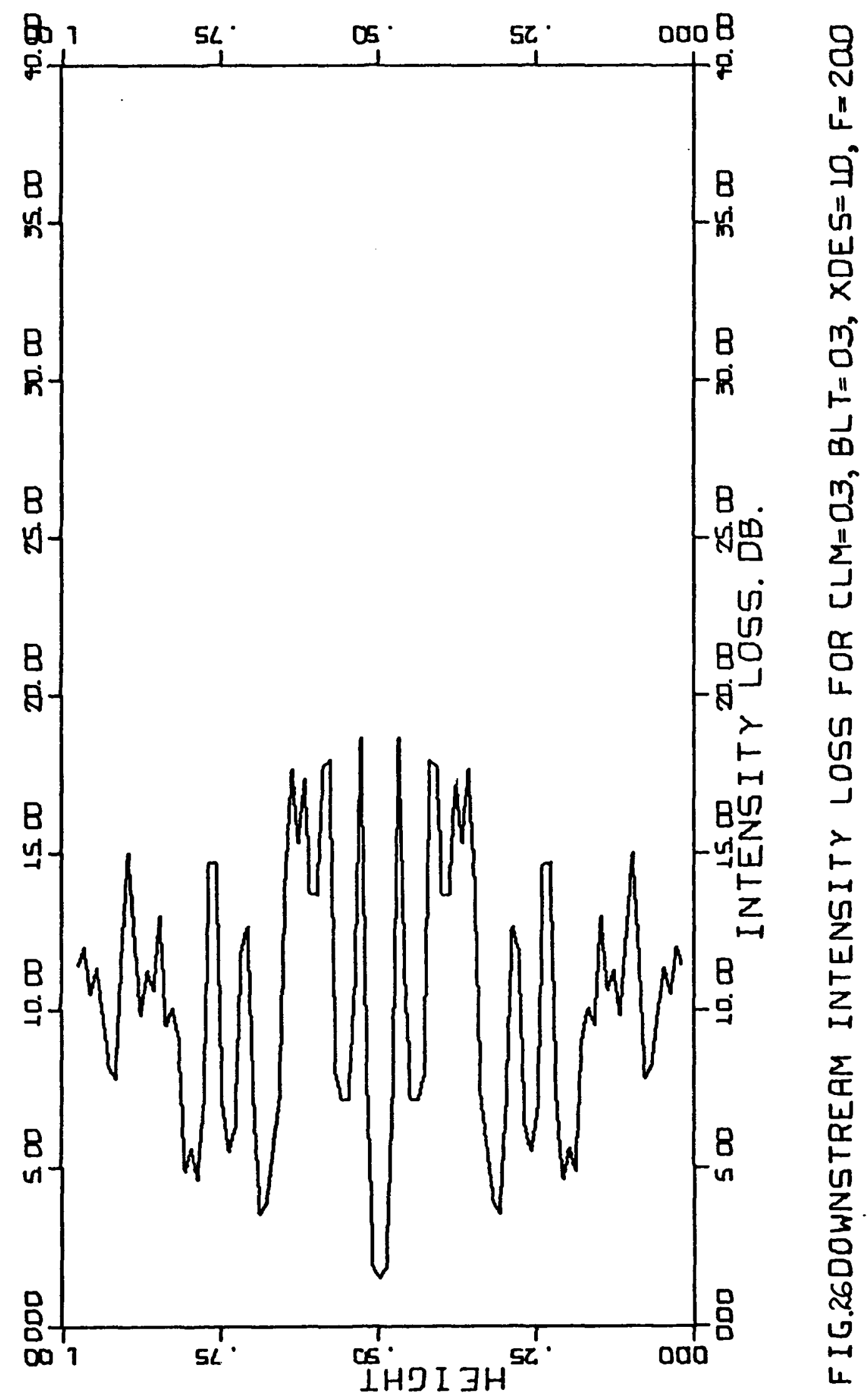




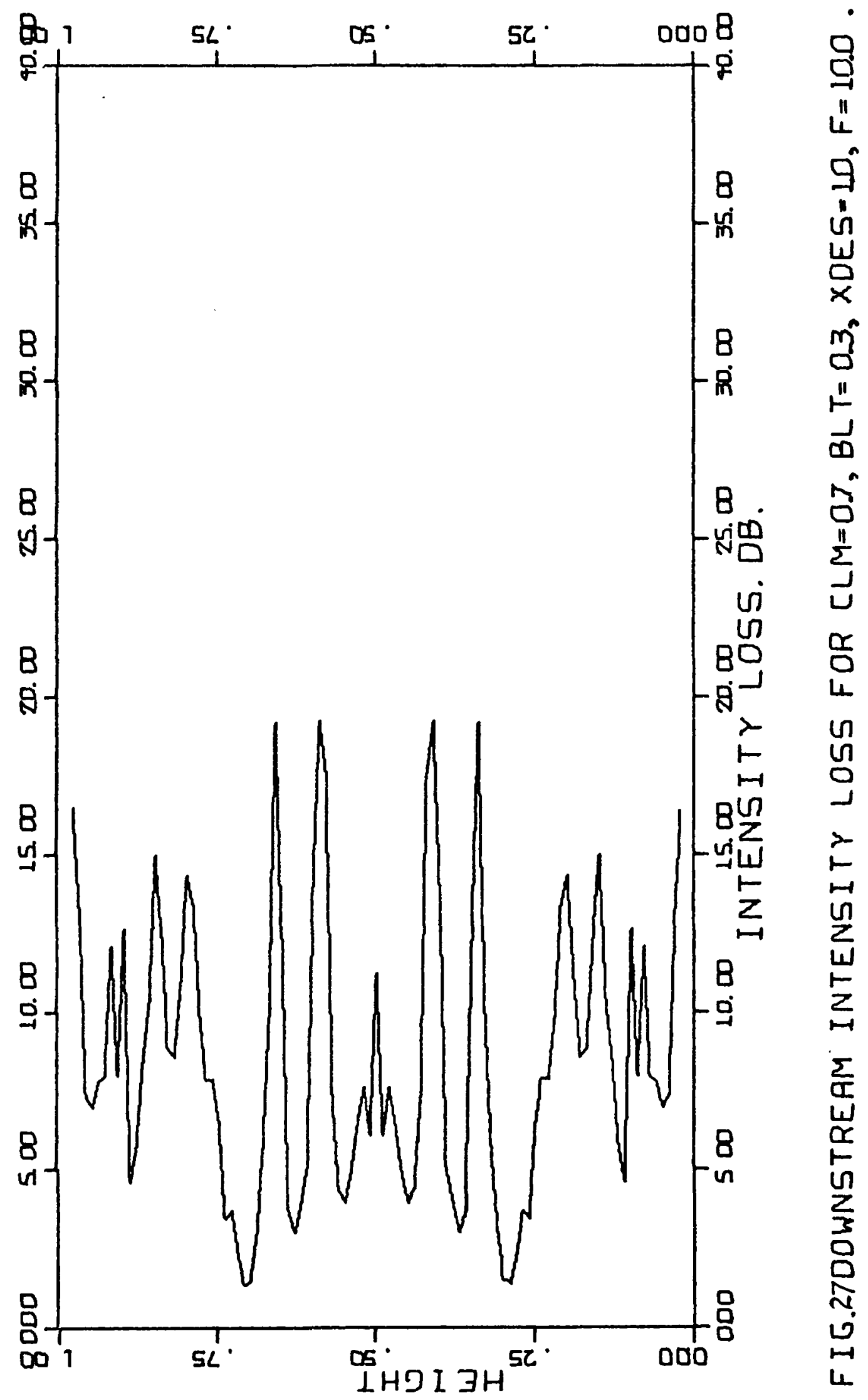




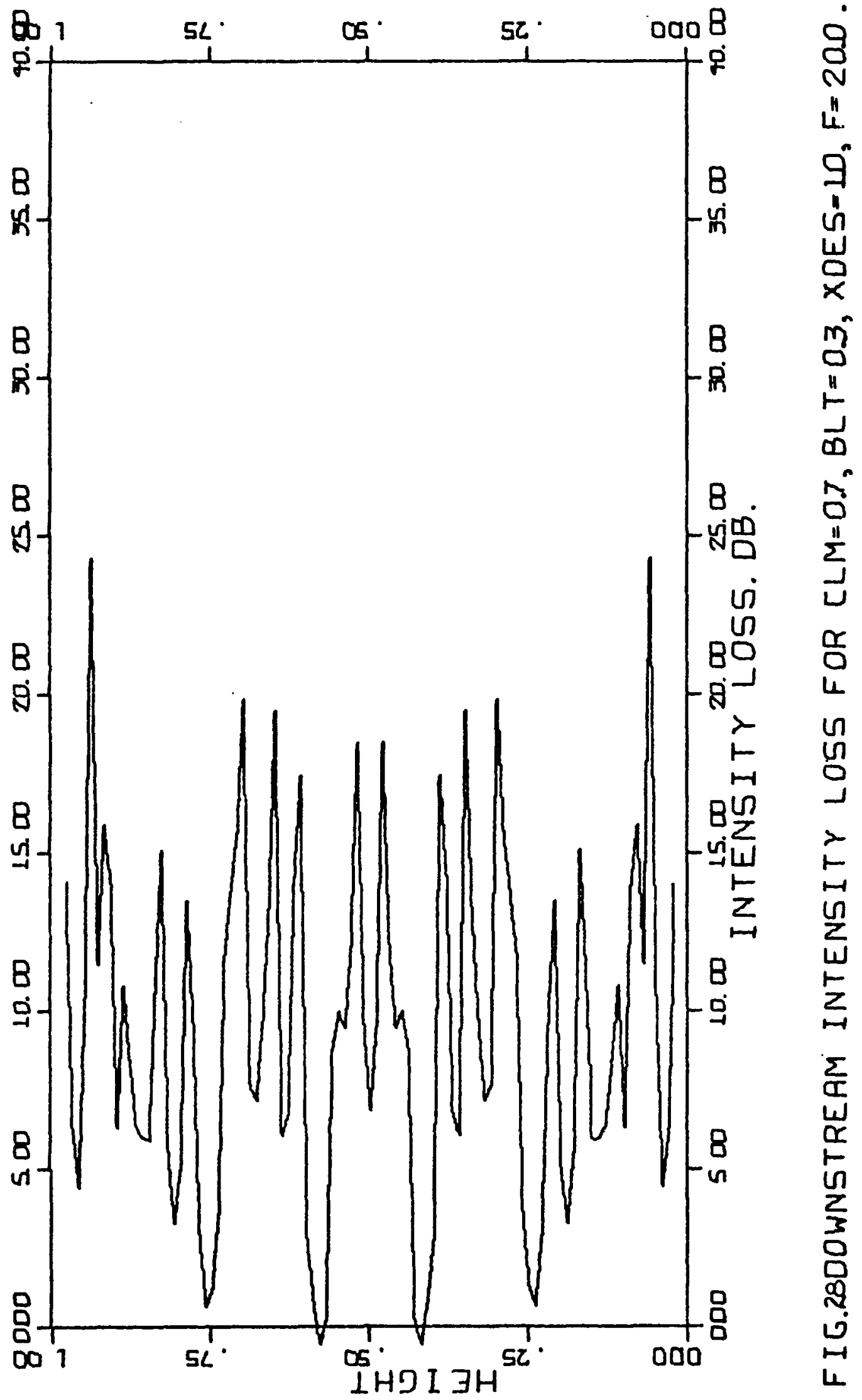




\section{CONCLUSIONS}

Through the application of ray acoustics, it was possible to model the propagation of sound through a hard walled duct and to study the effects of a sheared flow on the sound field within the duct. It was found that the ray plots could provide an excellent physical representation of the sound field by showing the paths of energy flow, and that the intensity loss plots could be used to show the distribution of acoustic energy at specified cross sections of a duct. The linearized ray approximation to the generalized wave equation was applied with little difficulty to a variety of flow velocity and flow gradient situations, except for upstream propagation where the theory broke down near the formation of a caustic.

Two phenomena were found which affected the sound field as a result of the flowing medium. First, the effects of refraction were found to result from variations in the wave phase velocity encountered as the sound propagated through regions of changing flow velocity. These effects were found to be greater for upstream propagation where the rays bent very sharply towards the center of the duct, and less significant for downstream propagation where the rays were bent only slightly towards the walls of the duct. Second, convection effects of the flow were shown to cause the wave to travel faster for downstream propagation and slower for 
upstream propagation.

Also for downstream propagation, the intensity loss plots showed the standing wave pattern which resulted from interference between direct and reflected rays. The flow gradients were found to cause very little change in the distribution of energy across the duct for downstream propagation, but they did have the effect of altering the phase relationships of the combined rays.

The ray theory proved to be much simpler to apply than the normal mode theory to the duct problem, and it was found that ray theory gave results which could be substantiated in practice. 


\section{REFERENCES}

1. Ingard, U., "Influence of Fluid Motion Past a Plane Boundary on Sound Reflection, Absorption, and Transmission," J.Acoust. Soc. Am., vol. 31, 1959, pp. 1035-1036.

2. Tack, D. H. and R. F. Lambert, "Influence of Shear Flow on Sound Attenuation in Lined Ducts," J. Acoust. Soc. Am., vol. 38, 1965, pp. 655-666.

3. W. Eversman, "The Effect of Mach Number on the Tuning of an Acoustic Lining in a Flow Duct," J.Acoust. Soc. Am., vol. $48,1970, \mathrm{pp} .425-428$.

4. C. I. Morfey, "Rotating Pressure Patterns in Ducts: Their Generation and Transmission," J. Sound Vibration, vol. I, 1964, pp. 60-87.

5. P. E. Doak and P. G. Vaidya, "Attenuation of Plane Wave and Higher Order Mode Sound Fropagation in Lined Ducts," J. Sound Vibration, vol. 12, 1970, pp. 201-224.

6. D. C. Pridmore-Brown, "Sound Propagation in a Fluid Flowing Through an Attenuating Duct," J. Fluid Mech., vol. 4, 1958, pp. 393-406.

7. P. Mungur and G. M. L. Gladwell, "Acoustic Wave Propagation in a Sheared Fluid Contained in a Duct," J. Sound Vibration, vol. 9, 1969, pp. 28-48.

8. Mechel, F., P. Mertens, and W. Schilz, "Research on Sound Propagation in Sound - Absorbent Ducts with Superimposed Air Streams," AMRL Report No. TDR-62-140, vol. III, Wright Patterson Air Force Base, Ohio, December, 1962.

9. P. Mungur and H. E. Plumblee, "Propagation and Attenuation of Sound in a Soft-Walled Annular Duct Containing a Sheared Flow, " Presented at the NASA Basic Noise Research Conference on July 14-15, 1969.

10. Eversman, W. "Effect of Boundary-Layer on the Transmission and Attenuation of Sound in a Acoustically Treated Circular Duct," J. Acoust. Soc. Am., vol. 49, 1971, pp. 1372-1380.

11. Hersh, A.S. and I. Catton, "Effect of Shear Flow on Sound Propagation in Rectangular Ducts," A.S. Hersh, Bolt Beranek and Newman Inc., Van Nuys, California, 1970.

12. Savkar, S. D. , "Propagation of Sound in Ducts with Shear Flow," J. Acous. Soc. Am., vol. 19, 1971, pp. 355-372. 
13. Bergmann, P. G. and A. Yaspan, Physics of Sound in the Sea, Part $I$. New York: Gordon and Breach Science Publishers, 1968.

14. Officer, C. B., Introduction to the Theory of Sound Transmission. New York: McGraw-Hill Book Company, Inc., 1958.

15. Kerr, D. E., Propagation of Short Radio Waves. New York: McGraw-Hill Book Company, Inc., 1951.

16. Brekhovskikh, I. M., Waves in Layered Media. Translated from the Russian by David Lieberman. New York: Academic Press, 1960.

17. Hayes, Wallace D., Rudolph C. Haefeli, and H.E. Kulsrud, "Sonic Boom Propagation in a Stratified Atmosphere with Computer Program," NASA CR-1299, April 1969.

18. Buell, C.E., "Variability of Sound Propagation Prediction due to Atmospheric Variability," Final report by Roman Aircraft Corp., NASA CR-61160, Jan. 1967.

19. Rayleigh, Lord, The Theory of Sound, vol. II. Macmillian Co., 1878. Reprint of 2nd ed., New York: Dover Publications, 1945 .

20. Barton, E.H., "On the Refraction of Sound by Wind," Phil. Mag. (6th series), vol. 1, 1901, pp. 159-165.

21. Heller, G.S., "Propagation of Acoustic Discontinuities in an Inhomogeneous Moving Iiquid Medium," J. Acoust. Soc. Am., vol. 24, 1952, p. 456 .

22. Milne, E.A., "Sound Waves in the Atmosphere," Philosophical Magazine, vol. 42, 1921, p. 96.

23. Kornhauser, E.T., "Ray Theory for Moving Fluids," J. Acoust. Soc. Am., vol. 25, September 1953, pp. 945-949.

24. Emden, R., "Beitrağe zur Thermodynamik der Atmosphäre," Meteorol. Zeit., vol. 35, 1918, pp. 13-29.

25. Blokhintzev, D., "The Acoustics of an Inhomogeneous Moving Medium," translated by R.T. Beyer and D. Mintzer (RAG., Brown University, 1952), p. 34. NACA TM 1399, 1956.

26. Moler, C. B. and I. P. Solomon, "Use of Splines and Numerical Integration in Geometrical Acoustics," I. Acous. Soc. Am., vol. 48, no. 3(part 2), September 1970, pp. 739- 
27. Anderson, G., R. Gocht, and D. Sirota, J.Acoust. Soc. Am., vol. 36, 1964, pp. 140-145.

28. Ugincius, P., "Intensity Equations in Ray Acoustics. I," J. Acoust. Soc. Am., vol. 45, 1969, pp. 193-205.

29. Warfield, J.T. and M.J. Jacobson, "Invariance of Geometric Spreading Loss with Changes in Ray Parametrization, "J. Acoustic Soc. Am., vol. 50, 1971, pp. 342-347.

30. Britton, J. R., R. Ben Kriegh, and Leon W. Rutland. University Mathematics II . San Franciso and London: W. H. Freeman and Company, 1965, p. 231.

31. Pedersen, M. A., "Comparison of Experimental and Theoretical Image Interference in Deep-Water Acoustics," J. Acoust. Soc. Am., vol. 34, September 1962, p. 1198.

32. Bucker, H. P., "The Ray Sweep-Out Method," J. Acoustic Soc. Am., vol. 52, part 2, July 1972, pp. 283-286.

33. Sochs, D. A.: and A. Silbiger, "Focusing and Refraction of Harmonic Sound and Transient Pulses in Stratified Media," J. Acoust. Soc. Am., vol. 49, August 1970, pp. 824-838. 


\section{APPENDIX}

The equation for the conditions at a horizontal reflecting boundary for the spreading loss equations have been derived by Ugincius (28). The ray path was represented as a general function in terms of the initial wave normal angle $\theta_{0}$ and the arc length $s$. This function can be expressed implicitly as

$$
f=f\left(x\left(s, \theta_{0}\right), z\left(s, \theta_{0}\right)\right) \quad .
$$

In this study it was more convenient to use travel time rather than arc length as a ray parameter. Since $s=s\left(t, \theta_{0}\right)$, equation $A-I$ can be written in terms of $t$ and $\theta_{0}$ through a transformation of variables as

$$
f=f\left[x\left(s\left(t, \theta_{0}\right), \theta_{0}\right), z\left(s\left(t, \theta_{0}\right), \theta_{0}\right)\right]=f\left[u\left(t, \theta_{0}\right), v\left(t, \theta_{0}\right] \cdot(A-2)\right.
$$

where $u$ and $v$ are the transformed variables. Starting with this functional relationship for the ray path it was possible to duplicate the procedure used by Ugincius and derive reflection conditions for $U_{t}, V_{t}$, and $\Theta_{t}$. (Note: Capital letters indicate partial derivatives with respect to $\theta_{0}$, while the subscript shows the variable which is held fixed. Primes are used to indicate derivatives with respect to $t$ with $\theta_{0}$ held constant.) These conditions were found to be

$$
\Delta U_{t}=0 \quad \Delta v_{t}=-2 v_{t} \quad \Delta \Theta_{t}=2 \frac{\Delta \mu / \Delta z}{\mu}\left(u^{\prime} / v^{\prime}\right) v_{t}-\theta_{t} \quad \cdot(A-3)
$$

Equations $A-3$ have the same form as those derived by Ugincius for a ray path expressed in terms of $s$ and $\theta_{0}$. Therefore, the reflection conditions were considered scalar point functions which were completely independent of the ray parameters used. 


\section{The vita has been removed from the scanned document}




\title{
APPLICATION OF RAY ACOUSTICS TO THE PROPAGATION OF SOUND IN DUCTS CONTAINING SHEARED FIOWS
}

\author{
by \\ Denny Warren Grimm
}

\begin{abstract}
Ray acoustics has been applied to the propagation of sound waves in a homogeneous moving medium with sheared flow bounded by the hard walls of a duct. Differential equations describing the ray trajectories and spreading losses along each ray were developed and solved numerically for a range of centerline ilach numbers and shear boundary-layers. Results were obtained which show the effects of upstream and downstream sound propagation on the ray paths. A methnd was also presented which allows the calculation of intensity loss profiles at specified cross sections of the duct.
\end{abstract}

